\title{
Generation and Regeneration of Thymic Epithelial Cells
}

\author{
Abdullah S. Alawam, Graham Anderson* and Beth Lucas* \\ Institute for Immunology and Immunotherapy, University of Birmingham, Birmingham, United Kingdom
}

The thymus is unique in its ability to support the maturation of phenotypically and functionally distinct $T$ cell sub-lineages. Through its combined production of $\mathrm{MHC}$-restricted conventional $\mathrm{CD} 4^{+}$and $\mathrm{CD}^{+}$, and $\mathrm{Foxp}^{+}$regulatory $\mathrm{T}$ cells, as well as non-conventional CD1d-restricted iNKT cells and invariant $\gamma \delta T$ cells, the thymus represents an important orchestrator of immune system development and control. It is now clear that thymus function is largely determined by the availability of stromal microenvironments. These specialized areas emerge during thymus organogenesis and are maintained throughout life. They are formed from both epithelial and mesenchymal components, and collectively they support a stepwise program of thymocyte development. Of these stromal cells, cortical, and medullary thymic epithelial

OPEN ACCESS

Edited by:

Nicolai Stanislas Van Oers, University of Texas Southwestern Medical Center, United States

Reviewed by:

Nancy Ruth Manley,

University of Georgia, United States Hyun Park,

National Cancer Institute (NCl), United States

*Correspondence:

Graham Anderson g.anderson@bham.ac.uk Beth Lucas

b.lucas.1@bham.ac.uk

Specialty section:

This article was submitted to $T$ Cell Biology,

a section of the journal

Frontiers in Immunology

Received: 17 February 2020 Accepted: 15 April 2020 Published: 07 May 2020

Citation:

Alawam AS, Anderson G and Lucas B (2020) Generation and Regeneration of Thymic Epithelial Cells.

Front. Immunol. 11:858

doi: 10.3389/fimmu.2020.00858 cells represent functional components of thymic microenvironments in both the cortex and medulla. Importantly, a key feature of thymus function is that levels of $\mathrm{T}$ cell production are not constant throughout life. Here, multiple physiological factors including aging, stress and pregnancy can have either short- or long-term detrimental impact on rates of thymus function. Here, we summarize our current understanding of the development and function of thymic epithelial cells, and relate this to strategies to protect and/or restore thymic epithelial cell function for therapeutic benefit.

\footnotetext{
Keywords: thymus, thymic epithelial cell, thymic atrophy, regeneration, bone marrow transplant, immune reconstitution
}

\section{INTRODUCTION}

While the bone marrow represents a major site of hemopoiesis, including hemopoietic stem cell development and maintenance as well as B-cell development, the generation of $\alpha \beta T$ cells relies upon the exit of lymphoid progenitors from the bone marrow and their entry into the thymus. Here, blood-borne thymus colonizing cells undergo a complex differentiation program that includes lineage restriction, proliferation, and $\mathrm{T}$ cell receptor gene rearrangement and selection. This results in the generation of a pool of self-tolerant $\alpha \beta$ TCR-expressing $C D 4^{+}$and $C D 8^{+}$mature thymocytes that then leave the thymus and form the peripheral $\mathrm{T}$ cell pool (1-3). Critically, intrathymic $\mathrm{T}$ cell development is a non-cell autonomous process and requires continual input from highly heterogeneous stromal cell populations that collectively form intrathymic microenvironments. Of particular importance is that such microenvironments contain both cortical and medullary regions, each consisting of, and defined by, specialized epithelial cells with differing roles $(4,5)$. Cortical thymic epithelial cells (cTEC) are responsible for driving immature $\mathrm{CD} 4^{-} \mathrm{CD} 8^{-}$lymphoid progenitors toward the $\mathrm{T}$ cell lineage, and the subsequent positive selection of $\mathrm{CD} 4^{+} \mathrm{CD} 8^{+}$ thymocytes. In addition, and following developmental stages in the cortex, thymocytes migrate into medullary thymic areas, with medullary thymic epithelial cells (mTEC) attracting positively 
selected thymocytes and providing environmental cues which aid in self-tolerance mechanisms that include deletion of autoreactive thymocytes via negative selection and sublineage divergence for the generation of Foxp $3^{+} \mathrm{CD} 4^{+}$T-Regulatory (TReg) cells $(6,7)$. Based on this, current models of intrathymic $\alpha \beta$ T cell development center around a step-wise process in which sequential interactions with cTEC and then mTEC generate and shape the $\alpha \beta \mathrm{T}$ cell pool. Importantly, in addition to generating conventional and Foxp3 ${ }^{+}$T-Reg that express diverse TCR repertoires, the thymus also supports the development of innatelike $\mathrm{T}$ cell subsets that can be defined by their expression of restricted TCR repertoires. In the embryonic period, examples of this are the serial waves of invariant $\gamma \delta$-cells that seed specific peripheral tissues, while the post-natal generation of CD1drestricted invariant NKT cells demonstrates how the thymus supports the development of multiple $\mathrm{T}$ cell types throughout life (8).

While TEC populations are known to be key regulators of these distinct $\mathrm{T}$ cell development programs, recent studies have uncovered significant new TEC heterogeneity that must be considered in relation to our understanding of microenvironmental control of $\mathrm{T}$ cell development (9-11). Significantly, therapeutic interventions can also be detrimental to thymus function $(12,13)$. Such clinical treatments include ablative preconditioning used in the treatment of cancer, which then impairs $\mathrm{T}$ cell mediated immune reconstitution following bone marrow transplantation. Consequently, studying thymic epithelial cells in both health and disease states is important to understand how thymus function is controlled, and how it might be manipulated for therapeutic benefit. Recently, important advances have been made in understanding the biology of thymic epithelium, including the developmental pathways that give rise to distinct cortical and medullary epithelial lineages. Furthermore, there is progress in how newly identified heterogeneity in thymic epithelium may map to functional specialization in thymic microenvironments.

\section{LINEAGE SPECIFIC THYMIC EPITHELIAL CELLS}

\section{cTEC and cTEC Heterogeneity}

The thymus cortex, and in particular the cTEC that reside there, play multiple key roles in T-cell development. These events include pre-TCR mediated transition of $\mathrm{CD}^{-} \mathrm{CD} 8^{-}$ precursors to the $\mathrm{CD}^{+}{ }^{+} \mathrm{CD} 8^{+}$stage, and the positive selection of $\mathrm{CD}^{+} \mathrm{CD}^{+}$cells expressing $\alpha \beta \mathrm{TCR}$ capable of $\mathrm{MHC}$ recognition. Interestingly, interactions between CXCR4 and its ligand CXCL12, a chemokine expressed by CTEC, have been reported to play a role in the regulation of both these events (Table 1). For example, CXCR4-CXCL12 interactions regulate the intrathymic positioning of $\mathrm{T}$ cell progenitors (14) while the maturation of pre-TCR expressing $\mathrm{CD} 4^{-} \mathrm{CD} 8^{-}$progenitors requires CXCR4-CXCL12 to act in concert with Notch signaling in order to drive $\beta$-selection $(15,16)$. For later stages of thymocyte development, CXCL12 has recently been reported to act as a cortex retention factor for $\mathrm{CD} 4^{+} \mathrm{CD} 8^{+}$thymocytes, which may enable cells to stay within the thymic cortex in order to undergo correct maturational events, including positive selection (17). Interestingly, of relevance to these studies that indicate the importance of CXCL12-CXCR4, is analysis of the expression and distribution pattern of CXCL12. Thus, analysis of CXCL12 $2^{\text {dsRed }}$ knockin mice showed that CXCL12 is expressed by $\mathrm{Ly}^{+} 1^{+}$cTEC, and is distributed throughout the thymic cortex microenvironment (18). As such, it is not currently clear how such a broad expression pattern of CXCL12 might relate to the possibility that particular regions of the thymus cortex are specialized to support specific maturational events. Moreover, it is also important to note that deletion of CXCR4 expression using $\mathrm{CD} 4{ }^{\text {cre }}$, which selectively targets $\mathrm{CD} 4^{+} \mathrm{CD} 8{ }^{+}$thymocytes and their downstream products, did not alter thymocyte development, nor the intrathymic positioning of $\mathrm{CD} 4^{+} \mathrm{CD} 8^{+}, \mathrm{CD}^{+}$, and $\mathrm{CD} 8^{+}$thymocytes (18). Thus, at stages downstream of pre-TCR mediated events, it is currently still unclear to what extent CXCR4 plays an important role. Perhaps relevant to this, CCR9 is an additional chemokine receptor expressed by $\mathrm{CD} 4^{+} \mathrm{CD} 8^{+}$thymocytes which, via interplay with plexinD1-semaphorin3E interactions, has been reported to play a role in the intrathymic positioning of thymocytes (19). Whether such findings collectively indicate potential functional redundancy and/or hierarchy in chemokine receptors and ligands that regulate the cortex residency of $\mathrm{CD} 4^{+} \mathrm{CD} 8^{+}$requires further investigation.

Regarding the ability of cTEC to support MHC class I mediated positive selection of $\mathrm{CD}^{+} \mathrm{T}$ cells, processing and presentation of peptides associated with MHC-I molecules requires proteasomal degradation. The thymoproteasome is a unique type of proteasome expressed specifically by cTECs, the catalytic subunit of which is $\beta 5$ t (Table 1 ). Mice deficient in $\beta 5 \mathrm{t}$ have reduced positive selection of $\mathrm{CD}^{+}$thymocytes (20). cTEC restricted expression of $\beta 5 t$ is also seen within human TECs, interestingly however analysis of humans carrying mutations within PSMB11 has not revealed any adverse effects (21). Nevertheless, $\beta 5 t$ is a defining feature of cTEC that directly underpins at least part of their functional specialization for positive selection. Importantly, while $\beta 5 \mathrm{t}$ expression by cTEC in the adult thymus is an important defining feature of cTEC functionality, it is also expressed by immature TEC progenitors. This is perhaps most notable in analysis of the embryonic thymus, where fate mapping of $\beta 5 t$ expressing cells showed that mTEC, including the Aire ${ }^{+}$subset, are derived from $\beta 5 \mathrm{t}-$ expressing cells (22). Thus, while $\beta 5 t$ expression is unique to TEC, and underpins cTEC function, its expression is not exclusive to mature cTEC. Moreover, expression of $\beta 5 t$ by TEC progenitors is also relevant to understanding functional heterogeneity in pathways of TEC development. For example, it is not clear whether $\beta 5 \mathrm{t}^{+}$progenitors are capable of cTEC functions such as positive selection prior to their transition toward the mTEC lineage. In this scenario, the embryonic thymus may harbor TEC progenitors that go through serial progression development (23), in which $\beta 5 \mathrm{t}^{+}$TEC first function as cTEC, and then differentiate further toward mTEC. Alternatively, $\beta 5 t^{+}$ progenitors may not possess functional capabilities of cTEC, and represent functionally immature cells that serve as a source of 
TABLE 1 | Differential gene expression in cTEC and mTEC subsets.

\begin{tabular}{|c|c|c|c|c|}
\hline Molecule & Gene Name & Expression pattern & Reported functions in thymus & References \\
\hline CD205 & Ly75 & Broad within cTEC & Apoptotic cell clearance & $(105)$ \\
\hline$\beta 5 \mathrm{t}$ & Psmb11 & $\begin{array}{l}\text { Broad within cTEC, also TEC } \\
\text { progenitors }\end{array}$ & Thymoproteosome component, CD8 positive selection & $(31,71,106)$ \\
\hline PRSS16 & Prss16 & CTEC & Thymus specific serine protease, CD4 positive selection & $(107)$ \\
\hline Delta like 4 & $D \| 4$ & CTEC & Notch ligand, regulator of T-cell commitment and $\beta$-selection & $(108)$ \\
\hline CXCL12 & $\mathrm{CxCl} 12$ & Broad within cTEC & Chemokine ligand for $\mathrm{CXCR} 4$, regulation of $\beta$-selection & $(14-18)$ \\
\hline CCL25 & Ccl25 & cTEC and mTEC & $\begin{array}{l}\text { Chemokine ligand for CCR9, recruitment and positioning of T-cell } \\
\text { progenitors, regulator of } \mathrm{CD} 4^{+} \mathrm{CD}^{+} \text {thymocyte migration }\end{array}$ & $(95,109,110)$ \\
\hline CCRL1 & Ackr4 & cTEC and mTEC & $\begin{array}{l}\text { Atypical chemokine receptor, scavenging receptor for CCL19, CCL21, } \\
\text { CCL25 }\end{array}$ & $(111,112)$ \\
\hline LT $\beta R$ & Ltbr & cTEC and mTEC & $\begin{array}{l}\text { Ligand for lymphotoxin and light, regulator of mTEC development and } \\
\text { thymic endothelium development. No known role in cTEC }\end{array}$ & $(34,45,96,97,113,114)$ \\
\hline Aire & Aire & mTEChi & Tissue restricted antigen expression, tolerance & $(115,116)$ \\
\hline Fezf2 & Fezf2 & mTEC ${ }^{\text {hi }}$ and mTEC ${ }^{l o}$ & Tissue restricted antigen expression, tolerance & $(44,45)$ \\
\hline RANK & Tnfrsf11a & $\begin{array}{l}\text { mTEC } \\
\text { progenitors }\end{array}$ & mTEC development & $(32,33,38,40,41,117)$ \\
\hline OPG & Tnfrsf11b & mTEC hi & Negative regulator of $\mathrm{mTEC}$ & $(33,39,41)$ \\
\hline IL25 & 1125 & Thymic tuft cells & Regulation of intrathymic ILC and iNKT-cells & $(9,11)$ \\
\hline IL15 & $\| 15$ & mTEC ${ }^{\circ}$ & IL15 transpresentation, regulation of iNKT-cells & $(59,118)$ \\
\hline IL15R $\alpha$ & $\| 15 r a$ & mTEC ${ }^{l o}$ & IL15 transpresentation, regulation of iNKT-cells & $(59)$ \\
\hline IL7 & $\| 7$ & $\begin{array}{l}\text { CTEC and mTEC in adult } \\
\text { thymus, TEC progenitors in } \\
\text { embryonic thymus }\end{array}$ & T-cell progenitor proliferation & $(70)$ \\
\hline CCL21 & Ccl21a & mTEC & $\begin{array}{l}\text { Chemokine ligand for CCR7, regulator of cortex to medulla migration of } \\
\text { SP thymocytes }\end{array}$ & $(37,42)$ \\
\hline Relb & Relb & mTEC & mTEC progenitor development & $(32,119,120)$ \\
\hline SCF & Kitlg & cTEC & Maintenance of $\mathrm{T}$ cell progenitors & $(121,122)$ \\
\hline
\end{tabular}

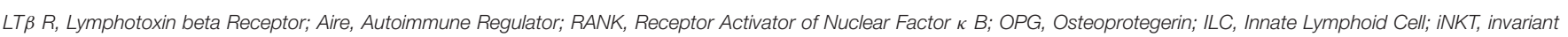
Natural Killer T Cell; SP, Single Positive; SCF, Stem Cell Factor.

functionally competent cTEC and mTEC. Whatever the case, expression of $\beta 5 t$ by both TEC progenitors and mature cTEC makes it difficult to directly define and discriminate between these cell types, particularly in the embryonic thymus.

Although the properties of the thymus cortex are becoming increasingly well-defined, functional heterogeneity within cTEC is still relatively poorly understood. Efforts have been made to investigate the cTEC population, and heterogeneity has been suggested using Sca-1 and $\alpha 6$-integrin, with Sca-1 positive cells expressing high levels of MHCII (20). Some of the difficulties in investigating the cTEC population is likely due at least to the relative low yield of cells obtained from the thymus compared to mTEC, making cTEC a difficult population to study. However, a recent study bypassed this problem by analyzing cTEC in mice in which CyclinD1 is overexpressed by $\mathrm{K} 5$ expressing cells. Although TEC were found at $\sim 100$-times greater frequency, the thymus from these mice had a normal structure, and supported a normal program of $\mathrm{T}$ cell development. Mass spectrometry proteomics and single cell RNA sequencing confirmed cTEC specific expression of Cathepsin L, TSPP, and $\beta 5 \mathrm{t}$, and mTECspecific expression of Cathepsin S, CD40, and Aire (24). These mice could prove to be a useful platform for further interrogation of the cTEC compartment.
In relation to functional heterogeneity within cTEC, thymic nurse cells (TNC) are large epithelial cell complexes in which single cTECs encase viable thymocytes. This is a unique feature of cTEC, and $\sim 10-15 \%$ of cTEC form such complexes; each containing between four and eight DP thymocytes (24). TNC are absent from the embryonic thymus, and are only detectable from 5 days post-birth; perhaps a reflection of a bias toward mature cTEC rather than cTEC-like progenitors. In addition, cTECs that form TNC have increased expression of CD205, CXCL12, TGF $\beta$, TSSP, and VCAM-1, compared to cTEC that are not part of TNC structures (25). Interestingly, thymocytes contained within TNC are enriched for those that have undergone secondary TCR $\alpha$ rearrangements, suggesting they may provide an environment then enables efficient positive selection. The presence of such epithelial-thymocyte complexes results in an estimated $20 \%$ of RNA isolated from total cTECs reflecting gene expression by enclosed DP thymocytes (24). This can be seen in single cell RNA sequencing of cTECs where newborn and adult cTECs appear to be contaminated with DP thymocytes (26). Recent single cell RNA sequencing analysis has begun to describe some heterogeneity within cTEC beyond TNC. For example, Bornstein et al. (9) found two clusters within cTEC with differential expression of genes including Dll4. 
Whether these cTEC differ in their functional capacity has not yet been tested.

Interestingly, constitutive autophagy is a feature of TECs, which contributes to the processing and presentation of MHCII associated peptides. Comparison of this process in cTEC and mTEC using GFP-LC3 transgenic mice to allow the detection of autophagosomes, showed that cTEC exhibit a higher frequency of autophagy-positive cells compared to mTECs (27). The function of this TEC specific feature is not clear, with conflicting reports in the literature. An initial study used $\operatorname{Atg} 5^{-1-}$ embryonic thymus lobes, grafted into nude mice, and showed that host mice generated symptoms of systemic autoimmunity (27). This was challenged by a later study, using targeted deletion of Atg7 in TECs using a K14 ${ }^{\text {Cre }}$ mouse line. These mice showed an absence of autoimmunity, even when aged (28). Cross comparisons are difficult between both studies due to the differing methods used to delete gene expression from TECs and analyze autoimmunity, thereby highlighting an area of research in need of further clarification.

\section{mTEC Stem Cells}

As TEC development involves the formation of distinct cTEC and MTEC populations (Figure 1), several studies have examined early timepoints in the development of these sublineages that are downstream of bipotent progenitors. For example, TEC expression of the tight junction components, Claudin-3 and Claudin-4 $(\mathrm{Cld} 3,4)$ has been reported to mark the emergence of the mTEC lineage, with cells expressing these markers giving rise to Aire $^{+}$mTEC (29). Moreover, a small population of TEC which co-express Cld3, 4 along with the stem cell marker SSEA-1 have been termed mTEC stem cells due to their selfrenewal capabilities and their ability to give rise to mTEC but not cTEC. mTEC stem cells have been further characterized by low expression of $\beta 5 t$ and CD205, and high expression of RANK and LT $\beta R$, and although they are capable of producing downstream mTEC populations in adult thymus, they do so with greatly reduced efficiency compared to in the embryonic thymus $(30,31)$. Despite expressing very low levels of $\beta 5$ t protein, fate-mapping experiments show these cells have a history of $\beta 5$ t expression, in keeping with them being downstream of $\beta 5 t^{+}$TEC progenitors (31). Interestingly, mice deficient in the master TEC transcription factor Foxn1 have normal frequencies of mTEC stem cells (32), suggesting that the defects in TEC development present in nude mice is downstream of these cells. Importantly, Baik et al. (32) also clarified the function of the TNFRSF member Relb during stages of mTEC development. Although $\mathrm{Relb}^{-/-}$mice had unaltered numbers of mTEC stem cells, using RANK ${ }^{\text {Venus }}$ reporter mice, it was shown that in the absence of Relb, Cld3, $4^{+}$mTEC fail to upregulate RANK expression. The importance of RANK signaling for the mTEC compartment is clear from the large reduction in mTEC, including Aire ${ }^{+}$mTEC in $\mathrm{Rankl}^{-/-}$ mice (33). Although the generation of mTEC stem cells does not require Foxn 1 or Relb, it has been shown at least in neonatal mice, to be partially dependent on LT $\beta \mathrm{R}$, as mTEC stem cell frequencies are reduced in neonatal $\mathrm{K} 14^{\mathrm{Cre}} \mathrm{LT} \beta \mathrm{R}^{\text {floxed }}$ mice where targeted deletion of LT $\beta$ R by TEC has been achieved (34). Despite the clear progress that has been made within this field, complex questions remain, which is due at least in part to a wide variety of markers used to identify TEC populations, as well as differing in vivo and in vitro methods used to assess their lineage potential. Further work is needed to build a more complete profile of relationships between mature TEC compartments and TEC progenitors, and the developmental requirements of each.

\section{Immature mTEC Progenitors}

In order to gain a better understanding of complexity within TEC populations, recent studies have interrogated the mTEC population using single cell RNA sequencing. One such study sorted total "unselected" mTECs, in addition to mTEC expressing specific Tissue Restricted Antigens (TRAs), namely Tspan8 and GP2 protein. To determine the likely developmental progression (10), clustering, and pseudotime trajectory analysis was performed on the single cell RNA sequencing data obtained from these populations. In agreement with other studies, this study highlighted a distinct population of mTEC phenotypically resembling jTECS (35) through their expression of Pdpn and lack of expression of Aire. Importantly, such cells were also defined by expression of the chemokine Ccl21a, that plays an important role in the recruitment of positively selected thymocytes into the medulla (Table 1). However, it is important to note that not all Ccl21 expressing mTEC appear to have high $P d p n$ expression (9). Interestingly, predicative analysis by Dhalla et al. (10) suggested CCL $21^{+} \mathrm{Pdpn}^{+}$immature mTEC follow a maturation pathway whereby they upregulate Aire expression, followed by expression of TRAs along with high levels of CD80 and CD86. Consistent with this, the gene signature associated with CCL $21^{+}$mTEC-I are present within the thymus at E14.5 whereas the genes relating to Aire ${ }^{+}$mTEC-II are not (9). More recent studies examining the developmental pathway of TEC development have used trajectory analysis of large data sets. Such analysis was performed on clusters of jTEC, mTEC ${ }^{\text {lo, }}$ and mTEC $^{\text {hi }}$, identified from single cell RNA sequencing data and supported the previously described immature phenotype of jTEC, and suggested they were most likely to become mTEC ${ }^{\text {hi }}$ before downregulating markers associated with maturation to become mTEC $^{\text {lo }}$ (36). While these studies provide important new information on mTEC heterogeneity, it is not fully clear whether CCL21-expressing mTEC, that typically lie within the $\mathrm{MHCII}^{\mathrm{lo}} \mathrm{CD} 80^{\mathrm{lo}}$ (mTEC ${ }^{\mathrm{lo}}$ ) compartment represent directly progenitors of later mTEC stages, including mTEC $^{\text {hi }}$. Indeed, although immature mTEC progenitors are known to reside within the bulk mTEC ${ }^{\text {lo }}$ compartment, the expression of CCL21 by some of these cells suggests that they are already functionally mature (37), and so could be defined as a mature mTEC subset. Perhaps relevant to this, at least in the embryonic thymus, mTEC progenitors that are able to give rise to Aire ${ }^{+}$ mTEC $^{\text {hi }}$ can be defined by their expression of RANK $(38,39)$ (Table 1). Indeed, in both embryonic and adult thymus, RANK itself is a key functional regulator of the maturation of mTEC progenitors into more mature mTEC $^{\text {hi }}(33,38-40)$. Importantly, while RANK expression has relevance to the study of mTEC progenitors, the nature of embryonic mTEC $^{\text {lo }}$ progenitors, and their full developmental potential, remains poorly understood. For example, it is not currently known whether $\mathrm{RANK}^{+}$ 


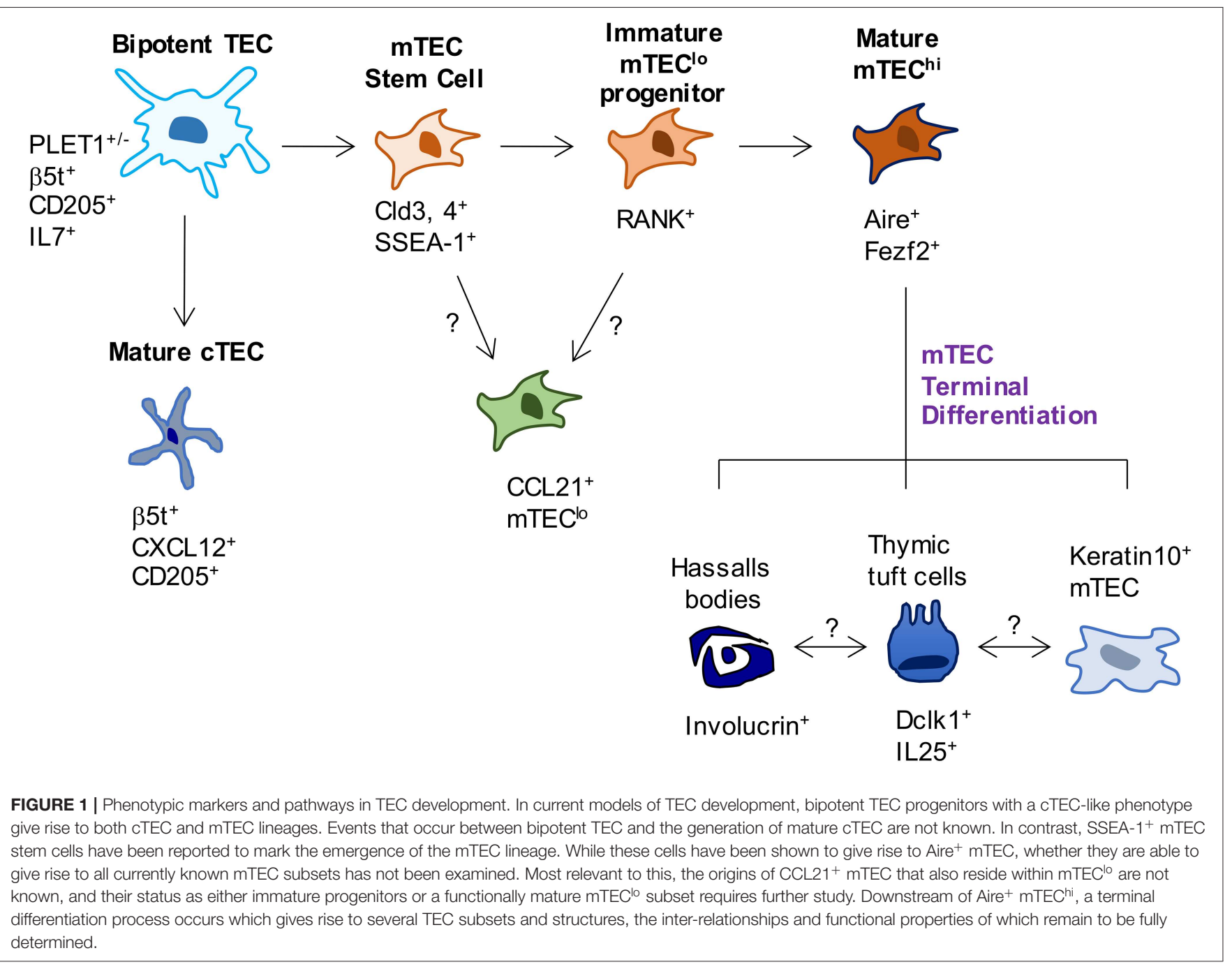

progenitors also express CCL21, a chemokine that is expressed by at least some mTEC (37) or whether $\mathrm{RANK}^{+}$progenitors can give rise to CCL $21^{+}$mTEC. Moreover, analysis of RANK ${ }^{\text {Venus }}$ reporter mice has shown that patterns of RANK expression in the adult thymus are complex, with multiple subsets of mTEC ${ }^{\text {lo }}$ and mTEC $^{\text {hi }}$, including CCL $21^{+}$cells and Aire ${ }^{+}$cells, demonstrating heterogeneity with regard to RANK expression (41). Thus, while it is clear that RANK is expressed by at least some mTEC progenitors, it is not known whether such progenitors have the potential to generate all mTEC subsets. Moreover, RANK may also be expressed by, and operate on, mTEC at other developmental stages. The recent generation of CCL $21^{\text {tdTomato }}$ reporter mice (42) together with availability of RANK ${ }^{\text {Venus }}$ mice (41) offers a possible way to generate new dual mTEC reporter mouse strains to examine new precursor-product relationships within the mTEC lineage.

\section{MHCII ${ }^{\text {hi }} \mathrm{CD} 80^{\text {hi }}$ mTEC $^{\text {hi }}$ and Thymic Tolerance}

Clear heterogeneity within mTEC exists, however segregation into subsets based on phenotype, developmental requirements and function can make conclusions and cross comparisons challenging. Routinely, mTECs are broadly subdivided into mTEC $^{\text {lo }}$ and mTEC $^{\text {hi }}$ according to their levels of MHCII and CD80. Perhaps the most defining feature used to discriminate populations within mTEC $^{\text {hi }}$ is expression of the autoimmune regulator (Aire). The transcription regulator Aire is required for efficient promiscuous gene expression of TRAs by mTEC, which is vital for the deletion of self-reactive thymocytes. This contributes to the multi-organ autoimmunity in Aire deficient mice and Aire deficient (autoimmune polyendocrinopathycandidiasis-ectodermal dystrophy) patients. The function of Aire exceeds TRA expression. For example, Aire regulates the expression of XCL1 in mTEC; a chemokine important for the medullary localization of thymic DC and Treg generation. Mice deficient in XCL1 exhibit symptoms of autoimmunity, suggesting that Aire promotes central tolerance via multiple mechanisms (43).

Not all promiscuous gene expression is dependent on Aire, as TRA expression is evident in Aire ${ }^{-/-}$mice. The transcription factor Fezf2, also expressed by mTEC, has been reported to be required for the expression of some Aire-independent genes 
(44). Parallel to Aire, Fezf2 expression is observed within the mTEC $^{\text {hi }}$ population, and moreover immunofluorescent staining reveals co-expression of both Aire and Fezf2 by the same cell $(44,45)$. Such mTEC $^{\text {hi }}$ that express high levels of Aire, Fezf2, and molecules associated with antigen presentation, were also described in a recent single cell RNA dataset and termed mTEC-II (9). Interestingly, co-expression of Aire and Fezf2 can be seen in human mTEC $(44,46)$, and its expression by Aire $^{+}$mTEC may suggest that similar mechanisms underpin central tolerance in mouse and man. Importantly, Fezf2 expression has also been observed within mTEC $^{\text {lo }}$ (38), suggesting TRA expression is not restricted to mTEC $^{\text {hi }}$ cells.

\section{mTEC Terminal Differentiation and Post-Aire mTEC: Involucrin ${ }^{+}$Cells}

Aire $^{+}$mature mTEC were once considered to be at the final stages of their maturation, with Aire expression indicative of subsequent apoptosis (47). However, several lines of evidence now show Aire ${ }^{+}$mTEC can continue their development beyond phases of Aire expression, to become TEC expressing markers typical of terminally differentiated keratinocytes $(48,49)$. These cells form distinct structures within the thymus medulla termed Hassall's corpuscles, and can be identified based on their expression of keratin-10 and involucrin. TEC with this phenotype are likely to be downstream of Aire ${ }^{+}$mTEC based on the ontogenetic appearance of both subsets. Aire ${ }^{+}$mTEC appear first during ontogeny, and are dependent on RANKL provision by DP thymocytes. Subsequently a population of involucrin ${ }^{+}$ mTEC becomes visible, which require LT $\alpha$ expression by positively selected thymocytes for their development (50). This is supported from direct analysis of TEC developmental pathways and fate mapping experiments, which showed that Aire ${ }^{+}$cells can proceed to become Aire ${ }^{-}$cells with lower levels of MHCII (49). Further analysis of post-Aire mTEC shows they lack expression of CCL21 (51), making them distinct from other populations of MHCII ${ }^{\mathrm{lo}}$ mTEC.

Although the role of Hassall's corpuscles remains elusive, further characterization of the phenotype and function of these cells has been achieved in the human thymus, where these medullary structures are much more prominent. In addition to keratin-10 and involucrin, Hassall's corpuscles in human thymus express filaggrin (52), and thymic stromal lymphopoietin (TSLP) (53). Whilst filaggrin expression within the murine medulla has been demonstrated (54), whether this specifically marks post-Aire mTEC is unclear. Expression of TSLP by Hassall's corpuscles in the human thymus has been shown to induce expression of markers associated with DC activation, which was needed to generate thymic T-Reg (53). This finding was followed up in mice, where TSLPZsG reporter lines were used to describe an enrichment of involucrin gene expression within reporter ${ }^{+}$mTEC (55), however whether there is a role for TSLP expression by terminally differentiated mTEC in the generation of T-Reg in mouse thymus is unknown.

\section{mTEC Terminal Differentiation and Post-Aire mTEC: Thymic Tuft Cells}

A combination of fate mapping experiments and single cell RNA sequencing analysis from two independent groups suggests there are two main populations of post-Aire $\operatorname{mTEC}(9,11)$. One population is the Keratin- $10^{+}$involucrin $^{+}$mTEC discussed above, whereas the other is a distinct population of TEC which resemble tuft cells that have been described at mucosal sites. Tuft cells are a type of chemosensory epithelial cell, most studied for their role in controlling helminth infection via activation of ILC2. Comparison of cells isolated from different tissues showed that tuft cells from the thymus had the greatest number of differentially expressed genes compared to tuft cells from other sites (56). Despite these differences, tuft cells from intestinal and thymic tuft cells share similarities, e.g., expression of IL25, Trmp5, Dclk1, and IL17RB.

The functions of these newly defined TEC are yet to be thoroughly explored. Unlike intestinal tuft cells, thymic tuft cells express high levels of MHCII (11) perhaps indicating an active role in antigen presentation and thymic selection. Interestingly however, evidence from Miller et al. (11) indicated a role for thymic tuft cells in central tolerance, through their expression of the tuft cell specific gene IL25. Thus, transplantation of tuft cell deficient Pou $23^{-/-}$thymic lobes into nude mice, resulted in the generation of anti-IL25 antibodies upon immunization, suggesting that tuft cells may act as an important source of antigen within the thymus (11). Perhaps also relevant to possible functional significance of thymic tuft cells it is interesting to note that in the gut, activation of tuft cells can be mediated by the microbial metabolite succinate. However, expression of the succinate receptor Sucnr1 is higher in small intestinal tuft cells compared to thymic tuft cells (56), and it is currently unknown whether thymic tuft cells undergo activation, and if so, how this might occur. Initial reports also suggest thymic tuft cells are capable of regulating innate immune networks within the thymus. Pou2f3 is the master regulator of tuft cell development, and as such, Pou $2 \mathrm{f3}^{-/-}$mice have been used to begin to determine the role of thymic tuft cells, and both thymic ILC and iNKT cells have been examined in tuft cell deficient mice. Bornstein et al. (9) proposed that due to the restricted thymic expression of IL25 by tuft cells, hematopoietic cells expressing IL25R may be dysregulated. In keeping with this, increased frequencies of ILC2 were present in the thymus of Pou $23^{-/-}$mice, however whether this is linked to absence of IL25 is not known. Interestingly, analysis of ILC subsets during thymus ontogeny revealed dynamic changes in their makeup. For example, while ILC3 are dominant in the embryonic thymus, ILC2 dominate post-natally (57). The reasons for this developmental switch in intrathymic ILC frequency is not clear. However, it is interesting to note that like ILC2, tuft cells also emerge post-natally, which together with the localization of both cell types within the medulla, may further emphasize a potentially important link between these cell types. If thymic tuft cells are regulators of ILC2, any increase in the latter in tuft cell deficient Pou $2 \mathrm{f}^{-/-}$mice would indicate that tuft cell products may act to limit ILC2 proliferation and or survival. While 
such factors remain unknown, as is the functional relevance of tuft cell control of ILC2 availability, it is perhaps important to note that ILC2 represent an intrathymic source of IL13 (57), a cytokine ligand for the type 2 IL4R that has been shown to be an important regulator of thymus emigration (58). Whether tuft cells limit ILC2-derived IL13 availability that then influences rates of thymus exit, has not been addressed. In addition to tuft cell-ILC interactions, Miller et al. (11) also examined Pou $23^{-/-}$mice to assess the potential impact of tuft cells on iNKT cells that represent a non-conventional $\alpha \beta \mathrm{T}$-cell lineage that is restricted to the non-polymorphic MHC class I like molecule CD1d. In line with a requirement for mTEC in NKT-cell development (59), Pou $23^{-/-}$mice were reported to have decreased frequencies of Tbet ${ }^{+}$NKT1, PLZF ${ }^{+}$NKT2, and Ror $\gamma \mathrm{t}^{+}$NKT17 within the thymus (11). Interestingly, a reduced frequency of Treg progenitors was also seen in the thymus of both Pou $23^{-/-}$mice and iNKT-cell deficient $C d 1 d^{-/-}$mice (60). The cellular and molecular interactions that connect tuft cells and iNKT-cells to the intrathymic development of Treg requires further investigation. Finally, studies demonstrating links between tuft cells and iNKT-cells are important as they indicate the importance of mTEC heterogeneity extends beyond its influence on conventional $\alpha \beta \mathrm{T}$-cell development in the thymus. How tuft cells control distinct subsets of iNKT-cells is currently not known. Given the patterns of IL25R expression by iNKT-subsets (61), and the selective intrathymic production of IL25 by tuft cells, one possibility that requires further examination is that tuft cells directly regulate at least some iNKT subsets, including NKT1 and NKT17 cells that express IL25R, via their provision of IL25. Whether tuft cells and/or additional mTEC subsets have the ability to influence individual iNKT subsets also requires further investigation.

Significantly, while there is evidence for the existence of DCLK $^{+}$tuft cells within the human thymus (9), whether human and mouse thymic tuft cells express a similar array of receptors and secreted factors is currently unknown. This will be important to consider when the functions of thymic tuft cells are more fully understood. While immunofluorescence staining of mouse thymus sections showed that both Keratin $10^{+}$mTEC and tuft cells are in close proximity to one another (11), the developmental relationships between the two populations is not clear. Although both populations can transit through an Aireexpressing stage, fate mapping experiments showed this isn't a feature of all tuft cells (11). Moreover, the requirement for Aire in their development may differ, for example, Aire ${ }^{-/-}$mice show significantly reduced frequencies of Keratin- $10^{+} \operatorname{mTEC}(11,51)$, whereas tuft cells are present in equivalent numbers in Aire ${ }^{-/-}$ mice (11). The initial description of thymic tuft cells proposed Hipk2, an Aire binding partner, to be a molecular regulator of this population, and the generation of Foxn $1^{\text {Cre }} \mathrm{Hipk} 2^{\text {floxed }}$ mice revealed reduced frequencies of thymic tuft cells (11), however the mechanism behind this is unknown. An additional regulator of Keratin- $10^{+}$mTEC development is LT $\alpha$ from positively selected thymocytes, as these terminally differentiated mTEC were found in reduced frequencies in $\mathrm{Lta}^{-/}, \mathrm{Ltbr}^{-/-}$, and Zap70-/- mice (50). Whether lymphotoxin signaling is also a regulator of tuft cell development in the mTEC lineage is yet to be determined.

\section{COMMON ORIGINS OF CTEC AND MTEC}

\section{Bipotent TEC Progenitors}

Despite the differing roles of cTEC and mTEC in the adult thymus, their development begins in the embryonic thymus from a common bipotent precursor that gives rise to both lineages. Initial experiments using purified populations of TEC and antibodies against MTS20 and MTS24 showed that both cTEC and $\mathrm{mTEC}$ are generated from Placenta expressed transcript- $1^{+}$ $\left(\mathrm{PLET}^{+}\right)$TEC $(62,63)$. However, at this time it was unclear whether a bipotent progenitor or individual cTEC and mTECrestricted precursors were contained within this fraction. Direct evidence was subsequently demonstrated for the existence of a bipotent TEC progenitor in the embryonic thymus. This was shown by the microinjection of a single EpCAM1 ${ }^{+} \mathrm{YFP}^{+}$cell into a non-YFP embryonic thymus, which was then grafted under the kidney capsule of a wildtype (WT) mouse. These grafts contained both $\mathrm{Ly} 51^{+}$cTEC, and Keratin $-5^{+}$mTEC, each anatomically segregated into distinct compartments, thus demonstrating that one cell can produce both TEC lineages (64). Similar conclusions were drawn from an independent study published at the same time that used mice in which a mutant allele of Foxn 1 could be reverted to wildtype function in single cells at post-natal stages. Following spontaneous induction of Foxn1 gene expression in a single cell, mice were able to generate thymic tissue containing both cTEC and mTEC providing evidence that bipotent TEC progenitors are also present within the post-natal thymus (65). Although both of these studies highlighted the existence of a bipotent TEC progenitor, the phenotype of such cells remains elusive, despite attempts of further characterization. Using reaggregate thymic organ cultures (RTOC) with purified populations of TEC, Rossi et al. (66) confirmed bipotent TEC progenitor potential by MTS $24^{+}$ TEC, but in addition showed equivalent capabilities within the MTS24- fraction. These findings support the notion that bipotent TEC progenitors express PLET-1, but shows additional progenitors are also present within the embryonic thymus which lack PLET-1 expression. The bipotent potential of PLET-1 expressing TEC isolated from adult thymus has been also assessed by grafting RTOC into WT mice. These grafts showed that UEA$\mathrm{I}^{-}$Ly51 ${ }^{+}$PLET- $1^{+}$cells with high expression of MHCII can give rise to both cTEC and mTEC, and continue to do so up to 9 months later (67).

Beyond these original descriptions of bipotent TEC progenitors, several studies have searched for evidence that supports the existence of bipotent TEC progenitors in the post-natal and adult thymus. For example, studies using longterm BrdU retention (indicative of a quiescent state) in adult TECs revealed $\mathrm{MHCII}{ }^{\mathrm{lo}} \alpha 6^{+} \mathrm{Sca}-1^{+}$cells at the corticomedullary junction $(\mathrm{CMJ})$ could generate both cTEC and mTEC lineages in reaggregate grafting experiments (68). However, it is not clear whether bipotent TEC or lineage-specific precursors were contained within this fraction, and their low expression 
of MHCII makes this population distinct from the PLET$1^{+}$cells described previously (67). In the embryo, further characterization of bipotent TEC progenitors has been possible, and studies have collectively described a cTEC-like phenotype of such cells using a variety of methods. For example, embryonic mTEC were shown to arise from TEC expressing markers commonly associated with cTEC, e.g., CD205 (69) and IL7 (70). In addition, Ohigashi et al. (22) fate mapped $\beta 5 \mathrm{t}$ expression; the proteasome subunit expressed by cTEC but not mTEC, and showed that cells with a history of $\beta 5 t$ expression later bore hallmark features of mTEC, including Aire expression. In a subsequent study using inducible $\beta 5$ tCre GFP mice to fate-map cells at post-natal stages, Ohigashi et al. (31) showed $<5 \%$ of mTEC were labeled when mice were treated with doxycycline after 1 week of age, whereas doxycycline treatment from E0 labeled $\sim 80 \%$ of mTEC. These results indicate that post-natal mTEC are derived from cells which express $\beta 5 \mathrm{t}$ embryonically. The shared expression of several markers e.g., CD205, Ly51, $\beta 5 t$, between cTEC and bipotent TEC progenitors makes the respective populations difficult to distinguish. However recent data from embryonic and adult TEC suggests differences now exist, and RNA sequencing analysis shows that embryonic TEC are enriched for genes involved in cell cycling and have a downregulation of genes involved in antigen presentation $(9,26)$. Such studies may support future approaches to identify differentially expressed genes that help to define and isolate TEC and TEC progenitor subsets.

Additional studies have provided information regarding the anatomical positioning of TEC progenitors in the post-natal thymus. In agreement with the description of an early cTEC phenotype of TEC progenitors, a combination of inducible fate mapping and confetti mice showed clusters of post- $\beta 5 \mathrm{t}$ expressing TEC that were concentrated at the CMJ (jTEC), which progressed to become mTEC (71). In addition, single cell RNA sequencing datasets show populations that resemble the immature jTECS described here $(9,10)$. Combined, this data supports a model of serial progression, whereby bipotent precursors acquire traits usually associated with cTEC, before bearing hallmark features of mTEC. Interestingly, cells within the adult thymus resembling stem cells have been described, with Ucar et al. (72) identifying cells capable of forming spheroid colonies typical of cells with stem cell properties. Such colonies, termed thymospheres, lacked expression of EpCAM1 and Foxn1, and were shown to have the capacity to generate both cTEC and mTEC. A more recent study revisited this issue, including the nature of thymosphere forming cells, and showed using a combination of fate mapping mouse strains that thymosphere forming cells are not of epithelial cell origin. Instead, they show by fate-mapping $\mathrm{Wnt} 1^{\mathrm{Cre}+}$ cells, that thymosphere forming cells are neural crest derived, and such structures can incorporate bystander TECs, thereby producing the results seen in the initial study (73). As such, the possible presence and identity of TEC populations with clonal and self-renewing properties remains unclear, and further studies are required to examine the earliest stages in embryonic and post-natal TEC development that give rise to the continual generation of cTEC and mTEC lineages.

\section{FACTORS AFFECTING RATES OF THYMUS FUNCTION}

Both chronic and acute damage to the thymus have detrimental effects on its ability to support $\mathrm{T}$ cell development. In particular, changes that take place within thymic epithelial microenvironments result in a reduction in $\mathrm{T}$ cell production, and such events can take place in several ways. For example, both age-related thymic involution and therapeutic cytoablative treatments erode TEC microenvironments which then impair rates of thymocyte development. Importantly however, regeneration processes can occur within the thymus, and several efforts have been made to understand this process and to harness it for therapeutic benefit.

\section{Age Related Thymus Atrophy}

Natural thymic involution, that occurs as a result of aging, significantly reduces rates of thymic function across the life course. Analysis of recent thymic emigrants as a measure of thymic function in Rag2GFP mice highlights the constant decline in de novo $\mathrm{T}$ cell production during the first 5-6 months of life (74). Unfortunately, identification of newly produced $\mathrm{T}$ cells in humans is more challenging, and currently relies on the PCR quantitation of circular DNA by-products of TCR gene rearrangements termed $\mathrm{T}$ Cell Receptor Excision Circles (TRECs), in conjunction with surface markers including CD31 and CD103. Such analysis shows that similar to mice, thymus function in humans also declines with age (75-78), resulting in a pool of peripheral $\mathrm{T}$ cells in the elderly which is dominated by clonally expanded cells (79). This impacts on the ability of an aged immune system to respond to challenge such as infection and vaccination.

Age associated thymic atrophy has been well-studied in mice, and attempts have been made to understand the mechanisms behind this phenomenon. The recruitment of $\mathrm{T}$ cell precursors into the thymus occurs via blood vessels at the CMJ, and expression of key molecules, e.g., P-selectin and CCL25 are important in this recruitment process (80). Initial reports suggested that expression of these factors is unaffected in aged mice (81), however a more recent publication showed reduced expression of CCL25 in the thymus from aged mice (82). Despite these discrepancies, recruitment of progenitor cells into the thymus does not appear to be the causative factor behind age related thymus involution, as the ability of an aged thymus to recruit intravenous-injected lymphoid progenitors is equivalent to a young thymus (81). Moreover, intrathymic injection of $\mathrm{T}$ cell precursors into young and aged thymi show reduced $\mathrm{T}$ cell development within the aged thymus (83). Combined, this data suggests that recruitment of progenitors may not be a simple explanation for the limited thymopoiesis in aged mice, but rather that other environmental factors within the thymus likely influence T cell development and thymus cellularity.

Interestingly, analysis of the stromal compartment by immunofluorescence in aged mice showed a progressive loss of both $\mathrm{CD} 205^{+}$cTEC and UEA- ${ }^{+}$mTEC, resulting in epithelial "free" areas $(81,84,85)$. This loss of TEC in aged mice is perhaps caused by reduced levels of proliferation and increased apoptosis 
(47, 81). Moreover, aged thymi show increased expression of phosphorylated $\mathrm{H} 2 \mathrm{AX}$ and $\mathrm{p} 53$ binding protein; markers of DNA damage and cellular senescence (84), which could account for the reduced thymus function seen with increasing age. The mechanisms that control TEC proliferation in aged mice is not fully understood, however it is interesting to note that whilst 95\% of embryonic TECs express Foxn1, the frequency of Foxn1 expressing TECs decreases post-natally (86). In keeping with reduced Foxn 1 expression in thymi from older mice, cTEC from older mice show reduced expression of Foxn1 target genes, e.g., Dll4, Kitl, Cxcl12, all of which are important for early stages of T cell development (82). Genetic alterations to Foxn1 in various mouse lines have been used in attempts to understand how Foxn1 may impact age related thymus involution. For example, overexpression of Foxn1 in young mice results in delayed thymus involution (87), whereas reduced Foxn1 expression in young mice causes premature thymus involution (88). In addition, regeneration of thymus function in aged mice has been possible via the upregulation of Foxn1 expression by TEC (82). Interestingly, such studies show Foxn1 aids TEC maintenance and recovery by inducing proliferation of TEC subsets, including MHCII $^{\text {lo }}$ TEC that are known to include TEC progenitors.

Proliferation of TEC is required for normal thymus growth during ontogeny, and this high rate of TEC proliferation during early stages of organogenesis is dependent on the transcription factor Myc. Importantly, Myc expression by TEC is high in the embryo but undergoes subsequent downregulation which correlates with age. This important process limits the extent to which the thymus can grow, and therefore may contribute to agerelated thymus involution. Cowan et al. (26) induced transgenic expression of Myc by TEC, which maintained a fetal gene signature in adult TEC, and caused excessive TEC proliferation and thymus hyperplasia in adult mice, suggesting this could be a mechanism by which to reverse age related thymic atrophy.

\section{Recovery of Thymus Function During Immune Reconstitution}

Cytoablative therapies such as chemotherapy and radiotherapy, that are often used in conjunction with bone marrow transplant (BMT), cause apoptosis of radiosensitive cells including thymocytes and TEC (89). As a result, there is a diminished capacity for the generation of newly produced naïve $\mathrm{T}$ cells. The recovery of $\mathrm{T}$ cells in the periphery occurs via two mechanisms; homeostatic expansion of $\mathrm{T}$ cells contained within the graft, and the export of naïve $\mathrm{T}$ cells from the thymus (Figure 2). The type of conditioning regime along with patient age, is likely to fine-tune the mechanism by which the peripheral $\mathrm{T}$ cell pool is restored $(90,91)$. As in homeostatic conditions, the production of new $\mathrm{T}$ cells requires the recruitment of $\mathrm{T}$ cell progenitors to the thymus. Thymus reconstitution following BMT appears to be limited by the number of progenitor cells available within the circulation, as a positive correlation is seen between numbers of BM cells administered, and the frequency of donor-derived DP thymocytes $(92,93)$. For these reasons, most studies have examined the process of thymus homing following BMT.

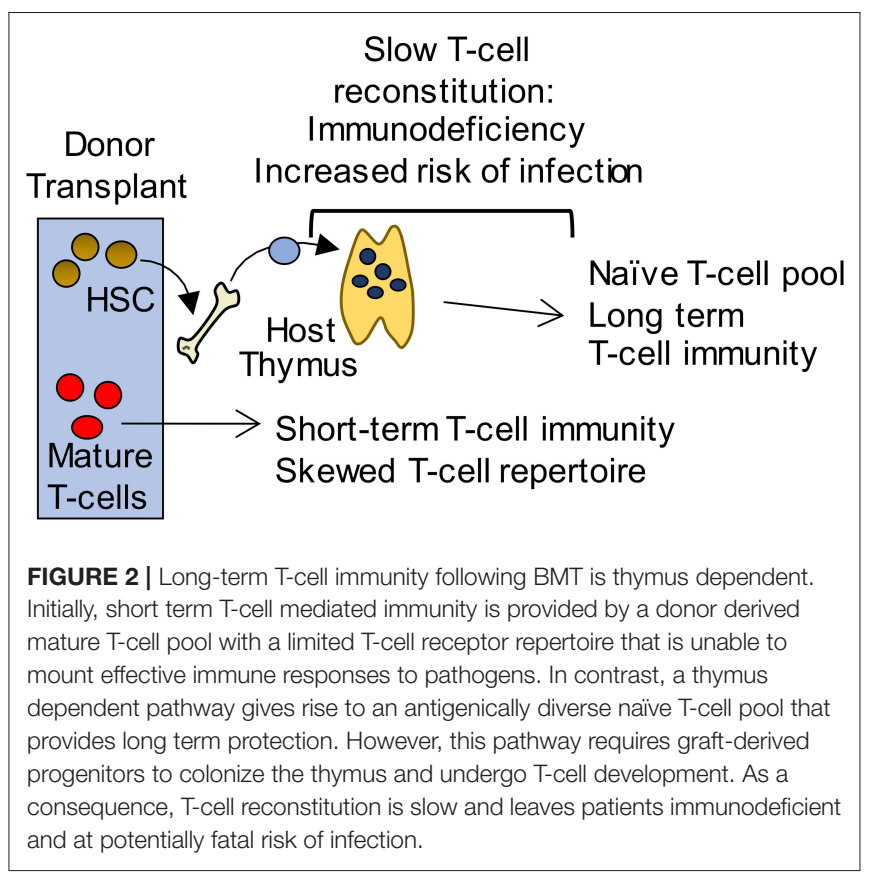

Thymic epithelial cells, including both cTEC and mTEC are reduced following irradiation in mouse models indicating the radiosensitive nature of these cells $(89,94)$. Although numbers of TEC are reduced, the ability of such cells to produce chemokines that are important $\mathrm{T}$ cell progenitor recruitment, such as CCL19, CCL21 and CCL25 is maintained following irradiation (89). In contrast, endothelial cells, which are important for the recruitment of $\mathrm{T}$ cell progenitors, appear to be radioresistant. Interestingly, the presentation of CCL 25 by thymic endothelium is transiently disrupted following irradiation which is proposed to limit thymus reconstitution, as pre-treatment of bone marrow cells with CCL25 caused increased $\mathrm{T}$ cell progenitor entry to the thymus following irradiation. This is in keeping with the requirement of CCR7 and CCR9 for $\mathrm{T}$ cell progenitor recruitment to the thymus during steady state conditions (95), which is mirrored at long-term time points following BMT where CCR7/CCR9 double-deficient bone marrow cells were shown to contribute very minimally to the pool of DP thymocytes. In contrast to this, early thymus reconstitution following BMT does not require CCR7 and CCR9, as T cell progenitors deficient in both chemokine receptors generate DP thymocytes to an equivalent ability to WT cells in a competitive bone marrow chimera model (92). Combined, these results indicate a transient period of time soon after BMT in which thymus settling occurs independently of CCR7 and CCR9. Furthermore, additional regulators of thymus homing in the steady state and following BMT have been identified, including PSGL1, the ligand for P-Selectin $(80,92)$, and lymphotoxin beta receptor (LT $\beta R$ ) (96). Within the thymus, LT $\beta$ R is expressed by TEC, thymic mesenchyme and thymic endothelial cells, and it's expression by endothelial cells is required for thymus homing during homeostatic conditions. This was illustrated by reduced frequencies of early thymic progenitors (ETP) in thymi from 
germline $\mathrm{Ltbr}^{-/-}$and Tek ${ }^{\mathrm{Cre}} \mathrm{LT} \mathrm{R}^{\text {floxed }}$ mice $(96,97)$. Moreover, the importance of LT $\beta R$ for thymus homing during BMT has been demonstrated by stimulating LT $\beta \mathrm{R}$ using an agonistic antibody during the time of BMT. This resulted in increased thymus and peripheral $\mathrm{T}$ cell reconstitution, suggesting that boosting thymus homing via this mechanism can favorably impact the peripheral $\mathrm{T}$ cell pool, and could thus pose a potential therapeutic target (96).

The impact of reduced thymus homing following irradiation is long-lived, as mice irradiated using a sublethal dose at 2 months of age, show reduced frequencies of ETP 16 months later (12). This study also showed unaltered expression of CCL25 following irradiation but did not assess CCL25 presentation by endothelial cells. Instead, Xiao et al. (12) propose that longterm effects are seen within LSK that reside within the BM. Quantitation of this population in irradiated mice revealed a decrease in the percentage and number of LSK 7 months post-irradiation, suggesting that reduced numbers of LSK are responsible for reduced ETP rather than reduced recruitment of these cells to the thymus. Importantly, this study showed that despite the reduction in ETP seen following BMT, there was a compensatory proliferation of DN3 thymocytes, and as such thymus cellularity was unaffected. Contradictory to this, Zhang et al. (89) used irradiation which was targeted to the upper or lower half of mice. Such treatment revealed an impact on donor-derived $\mathrm{T}$ cell development only when the upper half of the body was exposed to irradiation. Although this study didn't specifically target only the thymus with their method of irradiation, the results would suggest that damage to the thymus is the biggest driver in limiting $\mathrm{T}$ cell reconstitution following BMT.

\section{APPROACHES TO ENHANCE THYMUS RECOVERY}

\section{IL22 and BMP4}

While mechanisms that regulate thymic regeneration following damage remain unclear, several studies have provided some understanding of factors that may regulate this process. Following depletion of DP thymocytes, intrathymic IL-22 levels were found to increase, suggesting a link to mechanisms of endogenous recovery. In support of this, total body irradiation (TBI) of IL-22 deficient mice resulted in defective thymus regeneration compared to WT mice. Furthermore, when irradiation was targeted to the thymus, an increase in IL-22 was also observed suggesting a direct intrathymic recovery mechanism. Interestingly, IL-22 levels were recorded at the highest level when the thymus had the smallest cellularity, suggesting an inverse relationship between levels of IL-22 and thymic size. Production of IL-22 following damage was attributed to radioresistant thymic LTi/ILC3 which were present in increased numbers following thymic insult. Interestingly, irradiated $\mathrm{ROR} \gamma \mathrm{t}$ deficient mice that lack LTi/ILC3 did not increase their levels of intrathymic IL-22 after damage, suggesting a need for ROR $\gamma \mathrm{t}$-dependent cells, including LTi/ILC3, during thymus recovery following damage. Importantly, IL-22R is expressed by TEC, and IL-22 increased thymus cellularity due to increased proliferation of TEC and increased frequencies of all developing thymocyte subsets. These positive effects of IL-22 on thymus regeneration is limited to damage, as steady state mice treated with IL-22 showed no increase in total thymus cellularity (98). It is important to note that in this study, following TBI, LTi/ILC3 cells also upregulated RANKL expression, a molecule which has since been implicated in thymus regeneration. In another study (99), and following irradiation of WT mice, $\mathrm{CD}^{+} 5^{+}$cells upregulated RANKL expression compared to non-irradiated controls. Further analysis showed that RANKL expression was upregulated by radioresistant host LTi/ILC3 and $\mathrm{CD}^{+}$cells. Although LTi/ILC3 cell numbers in the thymus are low, their expression of RANKL was significantly higher than $\mathrm{CD} 4{ }^{+}$ SP thymocytes. To confirm the role of RANKL in thymus regeneration, WT mice were subjected to TBI, followed by neutralization of RANKL via antibody blocking that resulted in impaired TEC recovery. In a subsequent experiment, mice subjected to TBI and administered with exogenous RANKL showed significant increase in TECs compared to control mice. Here, exogenous RANKL treatment resulted in increased Ki67 expression by both CTEC and mTEC, indicative of increased proliferation, as well as reduced expression of pro-apoptotic genes. Interestingly, and perhaps in line with the potential importance of additional TNF Receptor superfamily members in thymus regeneration, a role for lymphotoxin signaling was also suggested, as stimulation by RANKL caused induction of LT $\alpha$ expression by LTi/ILC3 cells. Moreover, LT $\alpha$ deficient mice had reduced TEC recovery post-BMT compared to WT hosts, suggesting a mechanism of TEC recovery via LT $\alpha$ upregulation mediated by RANKL (99).

In addition to the potential of IL22, a recent study highlighted the involvement of bone morphogenic protein 4 (Bmp4) in thymus recovery following damage (100). Mice that were subjected to TBI show an upregulation of intrathymic Bmp4 levels suggesting this pathway may also be involved in thymus regeneration. In line with this, inhibition of Bmp4 by a pan BMP inhibitor prior to TBI caused an impairment in the thymus recovery mechanism. Bmp4 is expressed by multiple stromal cell types within the thymus, including fibroblasts and endothelial cells. Analysis of Bmp4 expression by qPCR on sorted populations of stromal cells following TBI revealed that Bmp4 expression was upregulated only by endothelial cells. Ex vivo expansion of EC that were transplanted post-TBI resulted in an increased TEC population, specifically cTEC, and qPCR analysis of cTEC showed an increase in Foxn1 levels as well as the Foxn1 target genes Dll4, Kitl, and Cxcl12, thus implicating Bmp4 by endothelial cells to initiate thymus recovery. Moreover, tamoxifen induced deletion of Bmp4 in endothelial cells prevented thymus recovery following TBI. Similar to the radioresistance of ILC3/LTi post-TBI, endothelial cells are also proposed to be radioresistant, as their frequencies remained unchanged in the thymus post-TBI. 


\section{Keratinocyte Growth Factor}

Thymic GVHD targets the thymic microenvironment and impairs thymopoiesis. However, studies have shown that thymic GVHD can be abrogated by keratinocyte growth factor (KGF) treatment prior to transplant $(94,101-103)$. In a model of GVHD, mice were transplanted with allogeneic splenocytes and treated with KGF for a period of 3 days prior to and after transplant. Control mice which did not receive KGF treatment showed a reduction in thymus weight and cellularity as a result of thymic GVHD. However, treatment with KGF inhibited the induction of thymic GVHD. Furthermore, overall percentage of donor $\mathrm{T}$ cells, specifically $\mathrm{CD}^{+} \mathrm{T}$ cells, infiltrating the thymus was shown to be reduced following KGF treatment. Despite this, absolute numbers of cells were not reduced suggesting that abrogation of thymic GVHD by KGF treatment was not due to decreased infiltration of donor transplanted $\mathrm{T}$ cells. Analysis of T cell development showed a loss of DP thymocytes in mice with GVHD, which was restored with KGF treatment. Although KGF treatment was able to protect the thymus from GVHD, it did not prevent donor $\mathrm{T}$ cell infiltration into the spleen which resulted in acute GVHD. The receptor for KGF (FGFR2IIIb) is expressed by TECs, thus the thymic microenvironment was analyzed post-GVHD induction. Thymic cortex/medulla organization was found to be severely disrupted, however organization was maintained following $\mathrm{KGF}$ treatment suggesting KGF acts on TEC to protect thymic microenvironments and subsequently promote thymopoiesis (101).

Other studies have also assessed the impact of KGF treatment in a mouse models that are aimed to mimic clinical settings. Here, mice were pre-conditioned with both irradiation and cyclophosphamide then reconstituted with MHC-mismatched bone marrow. Strikingly, KGF treatment allowed for sustained increased numbers of thymocytes for at least 3 months. In addition, KGF treatment increased frequencies of peripheral donor derived naive $\mathrm{T}$ cells, suggesting increased thymic output rather than peripheral T cell expansion (102). Furthermore, when KGF deficient $\left(F g f 7^{-/-}\right)$mice were sub-lethally irradiated to dissect the role of endogenous KGF on thymus regeneration, they displayed significant reductions in all thymocyte subsets. In addition, $\mathrm{Fgf7} 7^{-/-}$hosts that received allogeneic or syngeneic $\mathrm{BM}$ showed impaired regeneration of thymus as well as reduced peripheral donor and host $\mathrm{T}$ cells compared to WT hosts, suggesting host KGF is necessary to mediate thymus regeneration post-BMT (103). Similarly, Kelly et al. (94) studied effects of combined treatment of KGF and the p53 inhibitor Pifithrin$\beta$ (PFT- $\beta$ ) on thymus recovery. Lethally irradiated mice were reconstituted with $\mathrm{T}$ cell depleted $\mathrm{BM}$ and treated with KGF, PFT- $\beta$, or KGF and PFT- $\beta$. Analysis of the TEC compartment 2 weeks post-BMT showed improved thymus recovery in mice receiving combined treatment compared to either KGF or PFT$\beta$ treatment alone. Interestingly, TECs co-staining with Ly51 and Keratin5 were seen following combined treatment with KGF and PFT- $\beta$ suggesting bipotent progenitors may aid in the observed TEC regeneration. Importantly, intrathymic biotin labeling, as a means to measure thymic output, showed that combined treatment was able to improve thymic export (94). In relation to effects of KGF in humans, attempts to restore $\mathrm{T}$ cell numbers in relapse-remitting multiple sclerosis (RRMS) patients following antibody mediated-lymphocyte depletion, KGF treatment, given as palifermin, was shown to reduce thymopoiesis as $\mathrm{T}$ cell output was measured by naïve $\mathrm{T}$ cell count, RTE and TRECs. Strikingly, reduced thymic output was recorded 1 month post-palifermin administration, as numbers of naïve $\mathrm{CD}^{+}{ }^{+}$were reduced compared to the placebo group. In addition, frequencies of RTE were reduced following treatment with palifermin up to 6 months later. $\mathrm{CD}^{+}$effector memory cells were increased post-palifermin suggesting decrease in TCR repertoire (104). Despite improved thymopoiesis in murine models, KGF treatment in clinical trials has not improved $\mathrm{T}$ cell reconstitution, suggesting different requirements for KGF mediated TEC recovery in humans.

\section{CONCLUSIONS}

The importance of thymic epithelial microenvironments for $\mathrm{T}$ cell development is well-established. Despite this, we still lack a clear understanding of how TEC populations are established during development, and how they change during the lifecourse. Importantly, we still do not have a clear picture of the changes that take place in TEC populations in response to thymus damage, and how they are restored either naturally or following therapeutic intervention. As such, understanding the cellular makeup of TEC subsets is a key initial step to gain a clearer view of TEC biology, and also inform and improve approaches to manipulate TEC microenvironments with the longer-term goal of boosting immune system recovery. Relevant to this, several studies have now reported previously unrecognized heterogeneity within TEC compartments, and single cell RNA sequencing approaches represent a powerful approach to initially describe new TEC populations. A key goal of future studies will be to examine the potential functional importance of these newly described subsets, and to place them in a developmental sequence that will provide a detailed roadmap of stages in TEC development. For example, the mTEC $^{\text {lo }}$ population, that constitutes the majority of mTEC in the adult thymus, is now known to contain multiple populations that include progenitors of $\mathrm{mTEC}^{\mathrm{hi}}, \mathrm{CCL} 21^{+}$mTEC, and stages that represent post-mTEC ${ }^{\text {hi }}$ cells. Thus, and in contrast to initial thoughts, TTEC $^{\text {lo }}$ do not simply represent 'immature mTEC'. Approaches that enable the isolation and study of individual mTEC $^{\text {lo }}$ subsets will be needed to understand the functional importance of this diversity. Relevant to this, gaining a better understanding of the functional properties of recently described thymus tuft cells, that reside within mTEC $^{\text {lo }}$ and represent mTEC developmental stages that occur beyond the mTEC $^{\text {hi }}$ stage, may be important in revealing how the thymus medulla is able to support the development of multiple $\mathrm{T}$ cell lineages that include conventional $\alpha \beta$ T-cells, Foxp $3^{+}$T-Reg and CD1drestricted iNKT cells.

Finally, and as previously noted, while it is clear that TEC recovery occurs in damage settings that include bone 
marrow transplantation, it is not known whether such recovery involves a complete restoration of all TEC subsets that are now known to exist. Indeed, it is not known whether therapeutic treatments such as IL22, RANKL and KGF impart their effects via TEC progenitors, or via individual or multiple stages in the TEC developmental program. Again, understanding the relationships between newly described TEC populations, and identification of the factors that control their development, survival and expansion will be an important step in optimizing approaches to target TEC populations for therapeutic benefit.

\section{REFERENCES}

1. Kondo K, Takada K, Takahama Y. Antigen processing and presentation in the thymus: implications for T cell repertoire selection. Curr Opin Immunol. (2017) 46:53-7. doi: 10.1016/j.coi.2017.03.014

2. Klein L, Kyewski B, Allen PM, Hogquist KA. Positive and negative selection of the T cell repertoire: what thymocytes see (and don't see). Nat Rev Immunol. (2014) 14:377-91. doi: 10.1038/nri3667

3. Shah DK, Zuniga-Pflucker JC. An overview of the intrathymic intricacies of $\mathrm{T}$ cell development. J Immunol. (2014) 192:401723. doi: 10.4049/jimmunol.1302259

4. Takahama Y, Ohigashi I, Baik S, Anderson G. Generation of diversity in thymic epithelial cells. Nat Rev Immunol. (2017) 17:295-305. doi: 10.1038/nri.2017.12

5. Abramson J, Anderson G. Thymic epithelial cells. Annu Rev Immunol. (2017) 35:85-118. doi: 10.1146/annurev-immunol-051116-052320

6. Lio CW, Hsieh CS. A two-step process for thymic regulatory $\mathrm{T}$ cell development. Immunity. (2008) 28:10011. doi: 10.1016/j.immuni.2007.11.021

7. Tai X, Erman B, Alag A, Mu J, Kimura M, Katz G, et al. Foxp3 transcription factor is proapoptotic and lethal to developing regulatory $\mathrm{T}$ cells unless counterbalanced by cytokine survival signals. Immunity. (2013) 38:111628. doi: 10.1016/j.immuni.2013.02.022

8. Cowan JE, Jenkinson WE, Anderson G. Thymus medulla fosters generation of natural Treg cells, invariant $\gamma \delta \mathrm{T}$ cells, and invariant NKT cells: what we learn from intrathymic migration. Eur J Immunol. (2015) 45:65260. doi: 10.1002/eji.201445108

9. Bornstein C, Nevo S, Giladi A, Kadouri N, Pouzolles M, Gerbe F, et al. Singlecell mapping of the thymic stroma identifies IL-25-producing tuft epithelial cells. Nature. (2018) 559:622-6. doi: 10.1038/s41586-018-0346-1

10. Dhalla F, Baran-Gale J, Maio S, Chappell L, Hollander GA, Ponting CP. Biologically indeterminate yet ordered promiscuous gene expression in single medullary thymic epithelial cells. EMBO J. (2020) 39:e101828. doi: 10.15252/embj.2019101828

11. Miller CN, Proekt I, von Moltke J, Wells KL, Rajpurkar AR, Wang H, et al. Thymic tuft cells promote an IL-4-enriched medulla and shape thymocyte development. Nature. (2018) 559:627-31. doi: 10.1038/s41586-018-0345-2

12. Xiao S, Shterev ID, Zhang W, Young L, Shieh JH, Moore M, et al. Sublethal total body irradiation causes long-term deficits in thymus function by reducing lymphoid progenitors. J Immunol. (2017) 199:270112. doi: 10.4049/jimmunol.1600934

13. Ito R, Hale LP, Geyer SM, Li J, Sornborger A, Kajimura J, et al. Late effects of exposure to ionizing radiation and age on human thymus morphology and function. Radiat Res. (2017) 187:589-98. doi: 10.1667/RR4554.1

14. Plotkin J, Prockop SE, Lepique A, Petrie HT. Critical role for CXCR4 signaling in progenitor localization and $\mathrm{T}$ cell differentiation in the postnatal thymus. J Immunol. (2003) 171:4521-7. doi: 10.4049/jimmunol.171.9.4521

15. Janas ML, Varano G, Gudmundsson $K$, Noda M, Nagasawa T, Turner M. Thymic development beyond $\beta$-selection requires phosphatidylinositol 3-kinase activation by CXCR4. J Exp Med. (2010) 207:247-61. doi: 10.1084/jem.20091430

\section{AUTHOR CONTRIBUTIONS}

$\mathrm{AA}$ and $\mathrm{BL}$ reviewed the literature. $\mathrm{AA}, \mathrm{BL}$, and GA wrote and edited the manuscript.

\section{FUNDING}

Work in the laboratory is funded by the Medical Research Council (MR/N000919/1) and The Wellcome Trust $(211944 / \mathrm{Z} / 18 / \mathrm{Z})$. AA receives a $\mathrm{PhD}$ studentship from the Royal Embassy of Saudi Arabia Cultural Bureau.

16. Trampont PC, Tosello-Trampont AC, Shen Y, Duley AK, Sutherland AE, Bender TP, et al. CXCR4 acts as a costimulator during thymic $\beta$-selection. Nat Immunol. (2010) 11:162-70. doi: 10.1038/ni.1830

17. Kadakia T, Tai X, Kruhlak M, Wisniewski J, Hwang IY, Roy S, et al. E-proteinregulated expression of CXCR4 adheres preselection thymocytes to the thymic cortex. J Exp Med. (2019) 216:1749-61. doi: 10.1084/jem.20182285

18. Lucas B, White AJ, Parnell SM, Henley PM, Jenkinson WE, Anderson G. Progressive changes in CXCR4 expression that define thymocyte positive selection are dispensable for both innate and conventional $\alpha \beta$ T-cell development. Sci Rep. (2017) 7:5068. doi: 10.1038/s41598-017-05182-7

19. Choi YI, Duke-Cohan JS, Ahmed WB, Handley MA, Mann F, Epstein JA, et al. PlexinD1 glycoprotein controls migration of positively selected thymocytes into the medulla. Immunity. (2008) 29:888-98. doi: 10.1016/j.immuni.2008.10.008

20. Nitta T, Murata S, Sasaki K, Fujii H, Ripen AM, Ishimaru N, et al. Thymoproteasome shapes immunocompetent repertoire of $\mathrm{CD}^{+} \mathrm{T}$ cells. Immunity. (2010) 32:29-40. doi: 10.1016/j.immuni.2009.10.009

21. Ohigashi I, Ohte Y, Setoh K, Nakase H, Maekawa A, Kiyonari H, et al. A human PSMB11 variant affects thymoproteasome processing and CD8 ${ }^{+} \mathrm{T}$ cell production. JCI Insight. (2017) 2:e93664. doi: 10.1172/jci.insight.93664

22. Ohigashi I, Zuklys S, Sakata M, Mayer CE, Zhanybekova S, Murata S, et al. Aire-expressing thymic medullary epithelial cells originate from $\beta 5 \mathrm{t}$ expressing progenitor cells. Proc Natl Acad Sci U S A. (2013) 110:988590. doi: 10.1073/pnas.1301799110

23. Alves NL, Takahama Y, Ohigashi I, Ribeiro AR, Baik S, Anderson G, et al. Serial progression of cortical and medullary thymic epithelial microenvironments. Eur J Immunol. (2014) 44:16-22. doi: 10.1002/eji.201344110

24. Ohigashi I, Tanaka Y, Kondo K, Fujimori S, Kondo H, Palin AC, et al. Transomics impact of thymoproteasome in cortical thymic epithelial cells. Cell Rep. (2019) 29:2901-16.e6. doi: 10.1016/j.celrep.2019.10.079

25. Nakagawa Y, Ohigashi I, Nitta T, Sakata M, Tanaka K, Murata S, et al. Thymic nurse cells provide microenvironment for secondary $\mathrm{T}$ cell receptor $\alpha$ rearrangement in cortical thymocytes. Proc Natl Acad Sci U S A. (2012) 109:20572-7. doi: 10.1073/pnas.1213069109

26. Cowan JE, Malin J, Zhao Y, Seedhom MO, Harly C, Ohigashi I, et al. Myc controls a distinct transcriptional program in fetal thymic epithelial cells that determines thymus growth. Nat Commun. (2019) 10:5498. doi: 10.1038/s41467-019-13465-y

27. Nedjic J, Aichinger M, Emmerich J, Mizushima N, Klein L. Autophagy in thymic epithelium shapes the T-cell repertoire and is essential for tolerance. Nature. (2008) 455:396-400. doi: 10.1038/nature 07208

28. Sukseree S, Mildner M, Rossiter H, Pammer J, Zhang CF, Watanapokasin $\mathrm{R}$, et al. Autophagy in the thymic epithelium is dispensable for the development of self-tolerance in a novel mouse model. PLoS One. (2012) 7:e38933. doi: 10.1371/journal.pone.0038933

29. Hamazaki Y, Fujita H, Kobayashi T, Choi Y, Scott HS, Matsumoto M, et al. Medullary thymic epithelial cells expressing Aire represent a unique lineage derived from cells expressing claudin. Nat Immunol. (2007) 8:30411. doi: $10.1038 /$ ni1438 
30. Sekai M, Hamazaki Y, Minato N. Medullary thymic epithelial stem cells maintain a functional thymus to ensure lifelong central $\mathrm{T}$ cell tolerance. Immunity. (2014) 41:753-61. doi: 10.1016/j.immuni.2014.10.011

31. Ohigashi I, Zuklys S, Sakata M, Mayer CE, Hamazaki Y, Minato N, et al. Adult thymic medullary epithelium is maintained and regenerated by lineagerestricted cells rather than bipotent progenitors. Cell Rep. (2015) 13:143243. doi: 10.1016/j.celrep.2015.10.012

32. Baik S, Sekai M, Hamazaki Y, Jenkinson WE, Anderson G. Relb acts downstream of medullary thymic epithelial stem cells and is essential for the emergence of $\mathrm{RANK}^{+}$medullary epithelial progenitors. Eur J Immunol. (2016) 46:857-62. doi: 10.1002/eji.201546253

33. Hikosaka Y, Nitta T, Ohigashi I, Yano K, Ishimaru N, Hayashi Y, et al. The cytokine RANKL produced by positively selected thymocytes fosters medullary thymic epithelial cells that express autoimmune regulator. Immunity. (2008) 29:438-50. doi: 10.1016/j.immuni.2008.06.018

34. Wu W, Shi Y, Xia H, Chai Q, Jin C, Ren B, et al. Epithelial LT $\beta$ R signaling controls the population size of the progenitors of medullary thymic epithelial cells in neonatal mice. Sci Rep. (2017) 7:44481. doi: 10.1038/srep44481

35. Onder L, Nindl V, Scandella E, Chai Q, Cheng HW, Caviezel-Firner S, et al. Alternative NF-кB signaling regulates mTEC differentiation from podoplanin-expressing precursors in the cortico-medullary junction. Eur J Immunol. (2015) 45:2218-31. doi: 10.1002/eji.201545677

36. Miragaia RJ, Zhang X, Gomes T, Svensson V, Ilicic T, Henriksson J, et al. Single-cell RNA-sequencing resolves self-antigen expression during mTEC development. Sci Rep. (2018) 8:685. doi: 10.1038/s41598-017-1 9100-4

37. Lkhagvasuren E, Sakata M, Ohigashi I, Takahama Y. Lymphotoxin $\beta$ receptor regulates the development of CCL21-expressing subset of postnatal medullary thymic epithelial cells. J Immunol. (2013) 190:51107. doi: 10.4049/jimmunol.1203203

38. Rossi SW, Kim MY, Leibbrandt A, Parnell SM, Jenkinson WE, Glanville SH, et al. RANK signals from $\mathrm{CD}^{+} 3^{-}$inducer cells regulate development of Aire-expressing epithelial cells in the thymic medulla. J Exp Med. (2007) 204:1267-72. doi: 10.1084/jem.20062497

39. Akiyama N, Shinzawa M, Miyauchi M, Yanai H, Tateishi R, Shimo Y, et al. Limitation of immune tolerance-inducing thymic epithelial cell development by Spi-B-mediated negative feedback regulation. J Exp Med. (2014) 211:2425-38. doi: 10.1084/jem.20141207

40. Akiyama T, Shimo Y, Yanai H, Qin J, Ohshima D, Maruyama Y, et al. The tumor necrosis factor family receptors RANK and CD40 cooperatively establish the thymic medullary microenvironment and self-tolerance. Immunity. (2008) 29:423-37. doi: 10.1016/j.immuni.2008.06.015

41. McCarthy NI, Cowan JE, Nakamura K, Bacon A, Baik S, White AJ, et al. Osteoprotegerin-mediated homeostasis of $\mathrm{Rank}^{+}$thymic epithelial cells does not limit Foxp3 ${ }^{+}$regulatory T cell development. J Immunol. (2015) 195:2675-82. doi: 10.4049/jimmunol.1501226

42. Kozai M, Kubo Y, Katakai T, Kondo H, Kiyonari H, Schaeuble K, et al. Essential role of CCL21 in establishment of central self-tolerance in T cells. $J$ Exp Med. (2017) 214:1925-35. doi: 10.1084/jem.20161864

43. Lei Y, Ripen AM, Ishimaru N, Ohigashi I, Nagasawa T, Jeker LT, et al. Airedependent production of XCL1 mediates medullary accumulation of thymic dendritic cells and contributes to regulatory T cell development. J Exp Med. (2011) 208:383-94. doi: 10.1084/jem.20102327

44. Takaba H, Morishita Y, Tomofuji Y, Danks L, Nitta T, Komatsu N, et al. Fezf2 orchestrates a thymic program of self-antigen expression for immune tolerance. Cell. (2015) 163:975-87. doi: 10.1016/j.cell.2015. 10.013

45. Cosway EJ, Lucas B, James KD, Parnell SM, Carvalho-Gaspar M, White AJ, et al. Redefining thymus medulla specialization for central tolerance. J Exp Med. (2017) 214:3183-95. doi: 10.1084/jem.20171000

46. Gabrielsen ISM, Helgeland H, Akselsen H, Aass HCD, Sundaram AYM, Snowhite IV, et al. Transcriptomes of antigen presenting cells in human thymus. PLoS One. (2019) 14:e0218858. doi: 10.1371/journal.pone.0218858

47. Gray D, Abramson J, Benoist C, Mathis D. Proliferative arrest and rapid turnover of thymic epithelial cells expressing Aire. J Exp Med. (2007) 204:2521-8. doi: 10.1084/jem.20070795

48. Yano M, Kuroda N, Han H, Meguro-Horike M, Nishikawa Y, Kiyonari H, et al. Aire controls the differentiation program of thymic epithelial cells in the medulla for the establishment of self-tolerance. J Exp Med. (2008) 205:2827-38. doi: 10.1084/jem.20080046

49. Nishikawa Y, Hirota F, Yano M, Kitajima H, Miyazaki J, Kawamoto H, et al. Biphasic Aire expression in early embryos and in medullary thymic epithelial cells before end-stage terminal differentiation. J Exp Med. (2010) 207:963-71. doi: 10.1084/jem.20092144

50. White AJ, Nakamura K, Jenkinson WE, Saini M, Sinclair C, Seddon B, et al. Lymphotoxin signals from positively selected thymocytes regulate the terminal differentiation of medullary thymic epithelial cells. J Immunol. (2010) 185:4769-76. doi: 10.4049/jimmunol.1002151

51. Wang X, Laan M, Bichele R, Kisand K, Scott HS, Peterson P. PostAire maturation of thymic medullary epithelial cells involves selective expression of keratinocyte-specific autoantigens. Front Immunol. (2012) 3:19. doi: 10.3389/fimmu.2012.00019

52. Hale LP, Markert ML. Corticosteroids regulate epithelial cell differentiation and Hassall body formation in the human thymus. J Immunol. (2004) 172:617-24. doi: 10.4049/jimmunol.172.1.617

53. Watanabe N, Hanabuchi S, Soumelis V, Yuan W, Ho S, de Waal Malefyt $\mathrm{R}$, et al. Human thymic stromal lymphopoietin promotes dendritic cellmediated CD4 ${ }^{+} \mathrm{T}$ cell homeostatic expansion. Nat Immunol. (2004) 5:42634. doi: 10.1038/ni1048

54. Jee MH, Johansen JD, Buus TB, Petersen TH, Gadsboll AO, Woetmann $\mathrm{A}$, et al. Increased production of IL-17A-producing $\gamma \delta \mathrm{T}$ cells in the thymus of filaggrin-deficient mice. Front Immunol. (2018) 9:988. doi: 10.3389/fimmu.2018.00988

55. Dewas C, Chen X, Honda T, Junttila I, Linton J, Udey MC, et al. TSLP expression: analysis with a ZsGreen TSLP reporter mouse. J Immunol. (2015) 194:1372-80. doi: 10.4049/jimmunol.1400519

56. Nadjsombati MS, McGinty JW, Lyons-Cohen MR, Jaffe JB, DiPeso L, Schneider C, et al. Detection of succinate by intestinal tuft cells triggers a type 2 innate immune circuit. Immunity. (2018) 49:33-41 e7. doi: 10.1016/j.immuni.2018.06.016

57. Jones R, Cosway EJ, Willis C, White AJ, Jenkinson WE, Fehling HJ, et al. Dynamic changes in intrathymic ILC populations during murine neonatal development. Eur J Immunol. (2018) 48:1481-91. doi: 10.1002/eji.201847511

58. White AJ, Baik S, Parnell SM, Holland AM, Brombacher F, Jenkinson WE, et al. A type 2 cytokine axis for thymus emigration. J Exp Med. (2017) 214:2205-16. doi: 10.1084/jem.20170271

59. White AJ, Jenkinson WE, Cowan JE, Parnell SM, Bacon A, Jones ND, et al. An essential role for medullary thymic epithelial cells during the intrathymic development of invariant NKT cells. J Immunol. (2014) 192:2659-66. doi: 10.4049/jimmunol.1303057

60. Owen DL, Mahmud SA, Sjaastad LE, Williams JB, Spanier JA, Simeonov DR, et al. Thymic regulatory $\mathrm{T}$ cells arise via two distinct developmental programs. Nat Immunol. (2019) 20:195-205. doi: 10.1038/s41590-018-0289-6

61. Lee YJ, Holzapfel KL, Zhu J, Jameson SC, Hogquist KA. Steady-state production of IL-4 modulates immunity in mouse strains and is determined by lineage diversity of iNKT cells. Nat Immunol. (2013) 14:114654. doi: 10.1038/ni.2731

62. Bennett AR, Farley A, Blair NF, Gordon J, Sharp L, Blackburn CC. Identification and characterization of thymic epithelial progenitor cells. Immunity. (2002) 16:803-14. doi: 10.1016/S1074-7613(02)00321-7

63. Gill J, Malin M, Hollander GA, Boyd R. Generation of a complete thymic microenvironment by MTS24 ${ }^{+}$thymic epithelial cells. Nat Immunol. (2002) 3:635-42. doi: 10.1038/ni812

64. Rossi SW, Jenkinson WE, Anderson G, Jenkinson EJ. Clonal analysis reveals a common progenitor for thymic cortical and medullary epithelium. Nature. (2006) 441:988-91. doi: 10.1038/nature04813

65. Bleul CC, Corbeaux T, Reuter A, Fisch P, Monting JS, Boehm T. Formation of a functional thymus initiated by a postnatal epithelial progenitor cell. Nature. (2006) 441:992-6. doi: 10.1038/nature04850

66. Rossi SW, Chidgey AP, Parnell SM, Jenkinson WE, Scott HS, Boyd RL, et al. Redefining epithelial progenitor potential in the developing thymus. Eur J Immunol. (2007) 37:2411-8. doi: 10.1002/eji.200737275

67. Ulyanchenko S, O’Neill KE, Medley T, Farley AM, Vaidya HJ, Cook AM, et al. Identification of a bipotent epithelial progenitor population in the adult thymus. Cell Rep. (2016) 14:2819-32. doi: 10.1016/j.celrep.2016.02.080 
68. Wong K, Lister NL, Barsanti M, Lim JM, Hammett MV, Khong $\mathrm{DM}$, et al. Multilineage potential and self-renewal define an epithelial progenitor cell population in the adult thymus. Cell Rep. (2014) 8:1198209. doi: 10.1016/j.celrep.2014.07.029

69. Baik S, Jenkinson EJ, Lane PJ, Anderson G, Jenkinson WE. Generation of both cortical and Aire $^{+}$medullary thymic epithelial compartments from CD205 ${ }^{+}$progenitors. Eur J Immunol. (2013) 43:589-94. doi: 10.1002/eji.201243209

70. Ribeiro AR, Rodrigues PM, Meireles C, Di Santo JP, Alves NL. Thymocyte selection regulates the homeostasis of IL-7expressing thymic cortical epithelial cells in vivo. J Immunol. (2013) 191:1200-9. doi: 10.4049/jimmunol.1203042

71. Mayer CE, Zuklys S, Zhanybekova S, Ohigashi I, Teh HY, Sansom $\mathrm{SN}$, et al. Dynamic spatio-temporal contribution of single $\beta 5 \mathrm{t}^{+}$cortical epithelial precursors to the thymus medulla. Eur J Immunol. (2016) 46:84656. doi: 10.1002/eji.201545995

72. Ucar A, Ucar O, Klug P, Matt S, Brunk F, Hofmann TG, et al. Adult thymus contains $\mathrm{FoxN1}^{-}$epithelial stem cells that are bipotent for medullary and cortical thymic epithelial lineages. Immunity. (2014) 41:25769. doi: 10.1016/j.immuni.2014.07.005

73. Sheridan JM, Keown A, Policheni A, Roesley SNA, Rivlin N, Kadouri N, et al. Thymospheres are formed by mesenchymal cells with the potential to generate adipocytes, but not epithelial cells. Cell Rep. (2017) 21:93442. doi: 10.1016/j.celrep.2017.09.090

74. Hale JS, Boursalian TE, Turk GL, Fink PJ. Thymic output in aged mice. Proc Natl Acad Sci U S A. (2006) 103:8447-52. doi: 10.1073/pnas.0601040103

75. Douek DC, McFarland RD, Keiser PH, Gage EA, Massey JM, Haynes BF, et al. Changes in thymic function with age and during the treatment of HIV infection. Nature. (1998) 396:690-5. doi: 10.1038/25374

76. Ye $\mathrm{P}$, Kirschner DE. Reevaluation of $\mathrm{T}$ cell receptor excision circles as a measure of human recent thymic emigrants. J Immunol. (2002) 168:496879. doi: 10.4049/jimmunol.168.10.4968

77. Kimmig S, Przybylski GK, Schmidt CA, Laurisch K, Mowes B, Radbruch $\mathrm{A}$, et al. Two subsets of naive $\mathrm{T}$ helper cells with distinct $\mathrm{T}$ cell receptor excision circle content in human adult peripheral blood. J Exp Med. (2002) 195:789-94. doi: 10.1084/jem.20011756

78. McFarland RD, Douek DC, Koup RA, Picker LJ. Identification of a human recent thymic emigrant phenotype. Proc Natl Acad Sci U S A. (2000) 97:4215-20. doi: 10.1073/pnas.070061597

79. Qi Q, Liu Y, Cheng Y, Glanville J, Zhang D, Lee JY, et al. Diversity and clonal selection in the human T-cell repertoire. Proc Natl Acad Sci U S A. (2014) 111:13139-44. doi: 10.1073/pnas.1409155111

80. Rossi FM, Corbel SY, Merzaban JS, Carlow DA, Gossens K, Duenas J, et al. Recruitment of adult thymic progenitors is regulated by P-selectin and its ligand PSGL-1. Nat Immunol. (2005) 6:626-34. doi: 10.1038/ni1203

81. Gui J, Zhu X, Dohkan J, Cheng L, Barnes PF, Su DM. The aged thymus shows normal recruitment of lymphohematopoietic progenitors but has defects in thymic epithelial cells. Int Immunol. (2007) 19:120111. doi: 10.1093/intimm/dxm095

82. Bredenkamp N, Nowell CS, Blackburn CC. Regeneration of the aged thymus by a single transcription factor. Development. (2014) 141:162737. doi: 10.1242/dev.103614

83. Zhu X, Gui J, Dohkan J, Cheng L, Barnes PF, Su DM. Lymphohematopoietic progenitors do not have a synchronized defect with age-related thymic involution. Aging Cell. (2007) 6:663-72. doi: 10.1111/j.1474-9726.2007.00325.x

84. Aw D, Silva AB, Maddick M, von Zglinicki T, Palmer DB. Architectural changes in the thymus of aging mice. Aging Cell. (2008) 7:15867. doi: 10.1111/j.1474-9726.2007.00365.x

85. Li L, Hsu HC, Grizzle WE, Stockard CR, Ho KJ, Lott P, et al. Cellular mechanism of thymic involution. Scand J Immunol. (2003) 57:41022. doi: 10.1046/j.1365-3083.2003.01206.x

86. Rode I, Martins VC, Kublbeck G, Maltry N, Tessmer C, Rodewald HR. Foxn1 protein expression in the developing, aging, regenerating thymus. J Immunol. (2015) 195:5678-87. doi: 10.4049/jimmunol.1502010

87. Zook EC, Krishack PA, Zhang S, Zeleznik-Le NJ, Firulli AB, Witte PL, et al. Overexpression of Foxn1 attenuates age-associated thymic involution and prevents the expansion of peripheral CD4 memory T cells. Blood. (2011) 118:5723-31. doi: 10.1182/blood-2011-03-342097

88. Chen L, Xiao S, Manley NR. Foxn1 is required to maintain the postnatal thymic microenvironment in a dosage-sensitive manner. Blood. (2009) 113:567-74. doi: 10.1182/blood-2008-05-156265

89. Zhang SL, Wang X, Manna S, Zlotoff DA, Bryson JL, Blazar BR, et al. Chemokine treatment rescues profound T-lineage progenitor homing defect after bone marrow transplant conditioning in mice. Blood. (2014) 124:296304. doi: 10.1182/blood-2014-01-552794

90. Weinberg K, Blazar BR, Wagner JE, Agura E, Hill BJ, Smogorzewska M, et al. Factors affecting thymic function after allogeneic hematopoietic stem cell transplantation. Blood. (2001) 97:1458-66. doi: 10.1182/blood.V97.5.1458

91. Mackall CL, Fleisher TA, Brown MR, Andrich MP, Chen CC, Feuerstein IM, et al. Age, thymopoiesis, and $\mathrm{CD}^{+}{ }^{+}$T-lymphocyte regeneration after intensive chemotherapy. $N$ Engl J Med. (1995) 332:143-9. doi: 10.1056/NEJM199501193320303

92. Zlotoff DA, Zhang SL, De Obaldia ME, Hess PR, Todd SP, Logan $\mathrm{TD}$, et al. Delivery of progenitors to the thymus limits T-lineage reconstitution after bone marrow transplantation. Blood. (2011) 118:196270. doi: 10.1182/blood-2010-12-324954

93. Frasca D, Guidi F, Arbitrio M, Pioli C, Poccia F, Cicconi R, et al. Hematopoietic reconstitution after lethal irradiation and bone marrow transplantation: effects of different hematopoietic cytokines on the recovery of thymus, spleen and blood cells. Bone Marrow Transplant. (2000) 25:42733. doi: 10.1038/sj.bmt.1702169

94. Kelly RM, Goren EM, Taylor PA, Mueller SN, Stefanski HE, Osborn MJ, et al. Short-term inhibition of p53 combined with keratinocyte growth factor improves thymic epithelial cell recovery and enhances Tcell reconstitution after murine bone marrow transplantation. Blood. (2010) 115:1088-97. doi: 10.1182/blood-2009-05-223198

95. Zlotoff DA, Sambandam A, Logan TD, Bell JJ, Schwarz BA, Bhandoola A. CCR7 and CCR9 together recruit hematopoietic progenitors to the adult thymus. Blood. (2010) 115:1897-905. doi: 10.1182/blood-2009-08-237784

96. Lucas B, James KD, Cosway EJ, Parnell SM, Tumanov AV, Ware CF, et al. Lymphotoxin $\beta$ receptor controls $\mathrm{T}$ cell progenitor entry to the thymus. $J$ Immunol. (2016) 197:2665-72. doi: 10.4049/jimmunol.1601189

97. Shi Y, Wu W, Chai Q, Li Q, Hou Y, Xia H, et al. LT $\beta$ R controls thymic portal endothelial cells for haematopoietic progenitor cell homing and T-cell regeneration. Nat Commun. (2016) 7:12369. doi: 10.1038/ncomms12369

98. Dudakov JA, Hanash AM, Jenq RR, Young LF, Ghosh A, Singer NV, et al. Interleukin-22 drives endogenous thymic regeneration in mice. Science. (2012) 336:91-5. doi: 10.1126/science.1218004

99. Lopes N, Vachon H, Marie J, Irla M. Administration of RANKL boosts thymic regeneration upon bone marrow transplantation. EMBO Mol Med. (2017) 9:835-51. doi: 10.15252/emmm.201607176

100. Wertheimer T, Velardi E, Tsai J, Cooper K, Xiao S, Kloss CC, et al. Production of BMP4 by endothelial cells is crucial for endogenous thymic regeneration. Sci Immunol. (2018) 3:eaal2736. doi: 10.1126/sciimmunol.aal2736

101. Rossi S, Blazar BR, Farrell CL, Danilenko DM, Lacey DL, Weinberg KI, et al. Keratinocyte growth factor preserves normal thymopoiesis and thymic microenvironment during experimental graft-versus-host disease. Blood. (2002) 100:682-91. doi: 10.1182/blood.V100.2.682

102. Min D, Taylor PA, Panoskaltsis-Mortari A, Chung B, Danilenko DM, Farrell C, et al. Protection from thymic epithelial cell injury by keratinocyte growth factor: a new approach to improve thymic and peripheral T-cell reconstitution after bone marrow transplantation. Blood. (2002) 99:4592600. doi: 10.1182/blood.V99.12.4592

103. Alpdogan O, Hubbard VM, Smith OM, Patel N, Lu S, Goldberg GL, et al. Keratinocyte growth factor (KGF) is required for postnatal thymic regeneration. Blood. (2006) 107:2453-60. doi: 10.1182/blood-2005-07-2831

104. Coles AJ, Azzopardi L, Kousin-Ezewu O, Mullay HK, Thompson SA, Jarvis L, et al. Keratinocyte growth factor impairs human thymic recovery from lymphopenia. JCI Insight. (2019) 5:e125377. doi: 10.1172/jci.insight. 125377

105. Jenkinson WE, Nakamura K, White AJ, Jenkinson EJ, Anderson G. Normal T cell selection occurs in CD205-deficient thymic microenvironments. PLoS One. (2012) 7:e53416. doi: 10.1371/journal.pone.0053416 
106. Murata S, Sasaki K, Kishimoto T, Niwa S, Hayashi H, Takahama Y, et al. Regulation of $\mathrm{CD}^{+} \mathrm{T}$ cell development by thymus-specific proteasomes. Science. (2007) 316:1349-53. doi: 10.1126/science.1141915

107. Gommeaux J, Gregoire C, Nguessan P, Richelme M, Malissen M, Guerder $\mathrm{S}$, et al. Thymus-specific serine protease regulates positive selection of a subset of $\mathrm{CD}^{+}$thymocytes. Eur J Immunol. (2009) 39:95664. doi: 10.1002/eji.200839175

108. Fiorini E, Ferrero I, Merck E, Favre S, Pierres M, Luther SA, et al. Cutting edge: thymic crosstalk regulates delta-like 4 expression on cortical epithelial cells. J Immunol. (2008) 181:8199-203. doi: 10.4049/jimmunol.181.12.8199

109. Liu C, Saito F, Liu Z, Lei Y, Uehara S, Love P, et al. Coordination between CCR7- and CCR9-mediated chemokine signals in prevascular fetal thymus colonization. Blood. (2006) 108:2531-9. doi: 10.1182/blood-2006-05-024190

110. Krueger A, Willenzon S, Lyszkiewicz M, Kremmer E, Forster R. CC chemokine receptor 7 and 9 double-deficient hematopoietic progenitors are severely impaired in seeding the adult thymus. Blood. (2010) 115:190612. doi: 10.1182/blood-2009-07-235721

111. Lucas B, White AJ, Ulvmar MH, Nibbs RJ, Sitnik KM, Agace WW, et al. CCRL1/ACKR4 is expressed in key thymic microenvironments but is dispensable for T lymphopoiesis at steady state in adult mice. Eur J Immunol. (2015) 45:574-83. doi: 10.1002/eji.201445015

112. Ribeiro AR, Meireles C, Rodrigues PM, Alves NL. Intermediate expression of CCRL1 reveals novel subpopulations of medullary thymic epithelial cells that emerge in the postnatal thymus. Eur J Immunol. (2014) 44:291824. doi: 10.1002/eji.201444585

113. Boehm T, Scheu S, Pfeffer K, Bleul CC. Thymic medullary epithelial cell differentiation, thymocyte emigration, and the control of autoimmunity require lympho-epithelial cross talk via LT $\beta$ R. J Exp Med. (2003) 198:75769. doi: 10.1084/jem.20030794

114. James KD, Cosway EJ, Lucas B, White AJ, Parnell SM, Carvalho-Gaspar M, et al. Endothelial cells act as gatekeepers for LT $\beta$ R-dependent thymocyte emigration. J Exp Med. (2018) 215:2984-93. doi: 10.1084/jem.20181345

115. Anderson MS, Venanzi ES, Klein L, Chen Z, Berzins SP, Turley SJ, et al. Projection of an immunological self shadow within the thymus by the Aire protein. Science. (2002) 298:1395-401. doi: 10.1126/science.1075958

116. Heino M, Peterson P, Kudoh J, Nagamine K, Lagerstedt A, Ovod V, et al. Autoimmune regulator is expressed in the cells regulating immune tolerance in thymus medulla. Biochem Biophys Res Commun. (1999) 257:8215. doi: 10.1006/bbrc. 1999.0308

117. Irla M, Guerri L, Guenot $\mathrm{J}$, Serge $\mathrm{A}$, Lantz O, Liston A, et al. Antigen recognition by autoreactive $\mathrm{CD} 4^{+}$thymocytes drives homeostasis of the thymic medulla. PLoS One. (2012) 7:e52591. doi: 10.1371/journal.pone.0052591

118. Cui G, Hara T, Simmons S, Wagatsuma K, Abe A, Miyachi H, et al. Characterization of the IL-15 niche in primary and secondary lymphoid organs in vivo. Proc Natl Acad Sci U S A. (2014) 111:191520. doi: 10.1073/pnas.1318281111

119. Burkly L, Hession C, Ogata L, Reilly C, Marconi LA, Olson D, et al. Expression of relB is required for the development of thymic medulla and dendritic cells. Nature. (1995) 373:531-6. doi: 10.1038/373531a0

120. Weih F, Carrasco D, Durham SK, Barton DS, Rizzo CA, Ryseck RP, et al. Multiorgan inflammation and hematopoietic abnormalities in mice with a targeted disruption of RelB, a member of the NF-кB/Rel family. Cell. (1995) 80:331-40. doi: 10.1016/0092-8674(95)90416-6

121. Buono M, Facchini R, Matsuoka S, Thongjuea S, Waithe D, Luis TC, et al. A dynamic niche provides Kit ligand in a stage-specific manner to the earliest thymocyte progenitors. Nat Cell Biol. (2016) 18:15767. doi: $10.1038 /$ ncb3299

122. Rodewald HR, Kretzschmar K, Swat W, Takeda S. Intrathymically expressed c-kit ligand (stem cell factor) is a major factor driving expansion of very immature thymocytes in vivo. Immunity. (1995) 3:3139. doi: 10.1016/1074-7613(95)90116-7

Conflict of Interest: The authors declare that the research was conducted in the absence of any commercial or financial relationships that could be construed as a potential conflict of interest.

Copyright (c) 2020 Alawam, Anderson and Lucas. This is an open-access article distributed under the terms of the Creative Commons Attribution License (CC BY). The use, distribution or reproduction in other forums is permitted, provided the original author(s) and the copyright owner(s) are credited and that the original publication in this journal is cited, in accordance with accepted academic practice. No use, distribution or reproduction is permitted which does not comply with these terms. 